\title{
Covariance Statistics of Turbulence Velocity Components for Wind Energy Conversion System Design-Homogeneous, Isotropic Case
}

G. H. Fichtl

September 1983

Prepared for Pacific Northwest Laboratory under Agreement B-29086-A-L

Pacific Northwest Laboratory Operated for the U.S. Department of Energy by Battelle Memorial Institute 


\title{
DISCLAIMER
}

This report was prepared as an account of work sponsored by an agency of the United States Government. Neither the United States Government nor any agency thereof, nor any of their employees, makes any warranty, express or implied, or assumes any legal liability or responsibility for the accuracy, completeness, or usefulness of any information, apparatus, product, or process disclosed, or represents that its use would not infringe privately owned rights. Reference herein to any specific commercial product, process, or service by trade name, trademark, manufacturer, or otherwise, does not necessarily constitute or imply its endorsement, recommendation, or favoring by the United States Government or any agency thereof. The views and opinions of authors expressed herein do not necessarily state or reflect those of the United States Government or any agency thereof.

\author{
PACIFIC NORTHWEST LABORATORY \\ operated by \\ BATTELLE \\ for the \\ UNITED STATES DEPARTMENT OF ENERGY \\ under Contract DE-AC06-76RLO 1830
}

\begin{tabular}{|c|c|}
\hline \multicolumn{2}{|c|}{ Printed in the United States of America } \\
\hline \multirow{2}{*}{\multicolumn{2}{|c|}{$\begin{array}{l}\text { Available from } \\
\text { National Technical Information Service }\end{array}$}} \\
\hline & \\
\hline \multirow{3}{*}{\multicolumn{2}{|c|}{$\begin{array}{l}\text { United States Department of Commerce } \\
5285 \text { Port Royal Road } \\
\text { Springfield, Virginia } 22161\end{array}$}} \\
\hline & \\
\hline & \\
\hline \multirow{2}{*}{\multicolumn{2}{|c|}{$\begin{array}{l}\text { NTIS Price Codes } \\
\text { Microfiche A01 }\end{array}$}} \\
\hline & \\
\hline \multicolumn{2}{|c|}{ Printed Copy } \\
\hline & Price \\
\hline Pages & Codes \\
\hline $001-025$ & $\mathrm{~A} 02$ \\
\hline 026-050 & $\mathrm{A} 03$ \\
\hline 051-075 & A04 \\
\hline 076-100 & A05 \\
\hline $101-125$ & A06 \\
\hline $126-150$ & A07 \\
\hline $151-175$ & $\mathrm{~A} 08$ \\
\hline $176-200$ & A09 \\
\hline $201-225$ & A010 \\
\hline $226-250$ & A011 \\
\hline $251-275$ & A 012 \\
\hline & \\
\hline
\end{tabular}


G. H. Ficht

7703 Oakridge Drive, SE

Huntsville, Alabama 35802

September 1983

Prepared for

Pacific Northwest Laboratory

under Agreement B-29086-A-L

of Contract DE-AC06-76RL0 1830

for the U. S. Department of Energy

Pacific Northwest Laboratory

Richland, Washington 99352 
, 
PREFACE

Wind turbines are nearly unique among machines in that they must be dynamic, light, and yet strong enough to withstand substantial and continuing turbulent wind force in the atmospheric boundary layer. A program to optimize the cost of energy generated under such stringent conditions requires attention to the effects of wind in considerable detail during the design, testing and operation of the wind turbine.

This report was prepared under the supervision of the Design and Performing Evaluation Group of the Wind Characteristics Program Element of the Federal Wind Energy Program. The group is responsible for characterizing atmospheric properties in ways that are useful for designing and analyzing wind turbine response. This research includes the following components:

1. Restructuring and interpreting published conventional meteorological data for application to Wind Energy Conversion Systems (WECS)

2. Developing new characterizations of wind properties.

3. Describing wind characteristics at each important, distinctly different class of wind turbine site.

The ultimate objective of technical reports from work done in the Wind Characteristics Program Element is to provide practical information in a form easily interpreted and used by the engineering designer of WECS. The reports may have several different orientations. For example, some reports contain meteorological, mathematical and/or measurement detail or elaboration that provide advanced understanding of wind characteristics. These reports will subsequently be condensed and combined with other related reports to provide a more practical and general reference. Other reports represent multidisciplinary efforts, e.g., of engineers, atmospheric scientists, physicists and mathemat- 
icians. Still other reports provide advanced understanding of wind characteristics and relevation of unsuspected features of machine-wind interactions that should be considered by the wind energy community in design of machines. By quickly making this information known to users, we anticipate that research and improvement of the information will occur, and that some potential problems of machine-wind interactions will be brought to the attention of designers. We expect that in some cases there will be a need for later reports to condense this information and incorporate related work into more practical reference documents.

In brief, a diversity of topics and their treatment ranging from practical engineering design curves to rather fuller scientific expositions of the subject matter are to be found in our publications. All reports are directed toward the ultimate, but not distant, goal of having accurate and representative characteristics of the wind-turbine layer of the atmosphere for use in the design and evaluation of performance of WECS.

This report gives a modern theoretical indication of the significance of rotational sampling of the wind by a wind turbine blade spinning about a horizontal axis. The description of the process in the atmosphere and the graphical presentation of the modification of wind spectra that may be expected are to verify mathematically for the design engineer the concept of frequency shifting. This concept has been described by Connell (1980) using rotationally composited winds measured at the Pacific Northwest Laboratory (PNL) vertical plane array and more recently by Connell (1981) by theory and new observations. The detailed mathematical derivations and generalizations in this report are intended for engineers and scientists who wish to apply such theory in its present or improved form to WECS design. Present indications are such that the additional measurement and theoretical work now taking place on the rotational sampling of the wind by wind turbines with either horizontal or vertical axes will provide a firm basis for engineering decisions that will lead to improved design of WECS. The theoretical work reported here provided independent evidence of the concept 
and of the magnitude of the effect observed in the wind measurements at PNL. This evidence was important before undertaking several studies now started, which should provide practical, quantitative information for use in design and lifetime assessment.

\section{REFERENCES}

1. Conne11, J. R. 1980. Turbulence Spectrum Observed by a Fast-Rotating Wind Turbine Blade. PNL-3426, Pacific Northwest Laboratory, Richland, Washington.

2. Connell, J. R. 1981. The Spectrum of Wind Speed Fluctuations Encountered by a Rotating Blade of a Wind Energy Conver sion System: Observations and Theory. PNL-4083, Pacific Northwest Laboratory, Richland, Washington. 
.

, 


\section{ACKNOWLEDGEMENTS}

The author thanks Drs. William C. Cliff, James R. Connell, J. Christopher Doran, and David Powell for their advice and valuable discussions relative to the accomplishment of the research described herein and the preparation of this document. 



\section{SUMMARY}

When designing a wind energy conversion system (WECS), it may be necessary to take into account the distribution of wind across the disc of rotation. The specific engineering applications include structural strength, fatigue, and control. This wind distribution consists of two parts, namely that associated with the mean wind profile and that associated with the turbulence velocity fluctuation field. The work reported herein is aimed at the latter, namely the distribution of turbulence velocity fluctuations across the WECS disk of rotation

A theory is developed for the two-point, two-time covariance matrix for turbulence velocity vector components for wind energy conversion system (WECS) design. The theory is developed for homogeneous and isotropic turbulence with the assumption that Taylor's hypothesis is valid. The Eulerian turbulence velocity vector field is expanded about the hub of the WECS. Formulae are developed for the turbulence velocity vector component covariance matrix following the WECS blade elements. It is shown that upon specification of the turbulence energy spectrum function and the WECS rotation rate, the two-point, two-time covariance matrix of the turbulent flow relative to the WECS blade elements is determined. This covariance matrix is represented as the sum of nonstationary and stationary contributions. Generalized power spectral methods are used to obtain two-point, double frequency power spectral density functions for the turbulent flow following the blade elements. The Dryden turbulence model is used to demonstrate the theory. A discussion of linear system response analysis is provided to show how the double frequency turbulence spectra might be used to calculate response spectra of a WECS to turbulent flow. Finally the spectrum of the component of turbulence normal to the WECS disc of rotation, followina the biade elements, is compared with experimental results. 


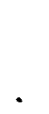




\section{TABLE OF CONTENTS}

PREFACE . . . . . . . . . . . . . . . . . . . . . . . . . . . . . . .

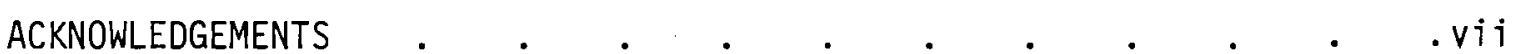

SUMMARY . . . . . . . . . . . . . . . . . . . . .

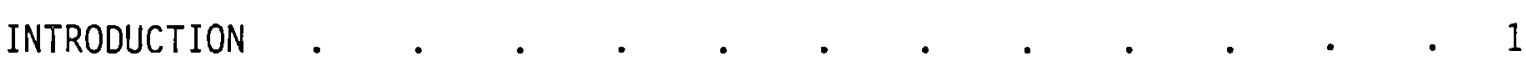

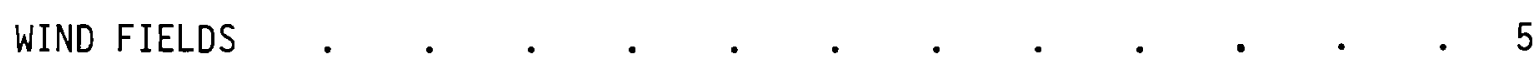

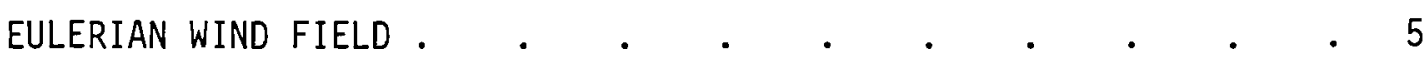

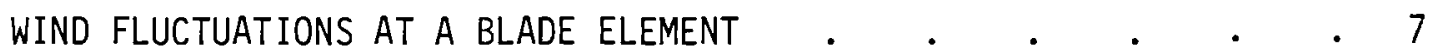

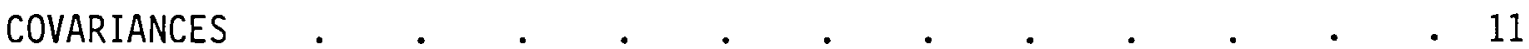

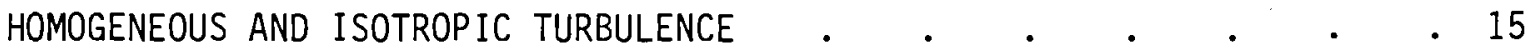

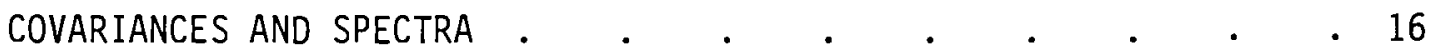

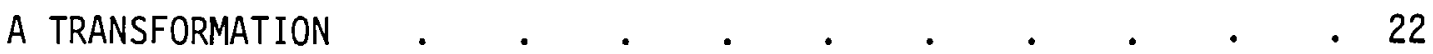

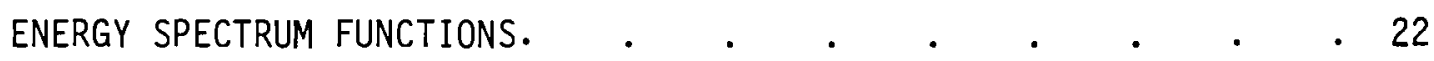

CALCULATION OF SPECTRAL FUNCTION

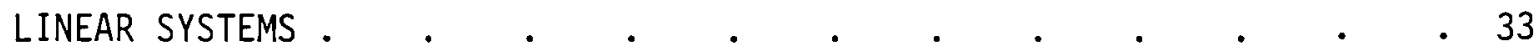

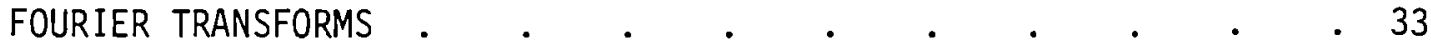

GENERALIZED TURBULENCE SPECTRAL DENSITIES • • • • • • • • 36

FREQUENCY-TIME SPECTRAL DENSITIES .

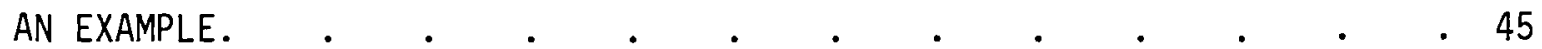

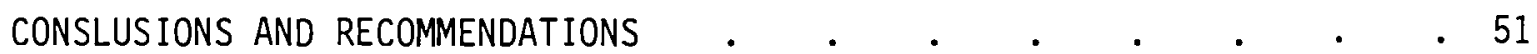

REFERENCES.

APPENDIX A - APPROACH FOR DIRECT CALCULATION OF TWO-TIME, TWO-POINT TURBULENCE STATISTICS . . . . . . . . . . A

APPENDIX B - FORMULATION OF THEORY FOR NONHOMOGENEOUS TURBULENT FIELD - B-1

APPENDIX C - SPECTRAL CONTRIBUTION RESULTING FROM MEAN FLOW SHEAR. - C C-1 


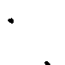




\section{INTRODUCTION}

The purpose of this document is to develop a model that can be used to estimate the two-point, two-time covariance statistics of atmospheric turbulence velocity fluctuations following the blade elements of a wind energy conversion system (WECS). The flow in the atmospheric boundary layer can be represented in terms of a mean wind field (obtained by averaging wind records over a one hour period, say) and a departure from this mean. The departures, termed turbulence velocity fluctuations, constitute a three-dimensional, time dependent stochastic field. The WECS blade elements experience a highly time-dependent flow as a result of these turbulence velocity fluctuations. Because of the stochastic nature of these turbulence velocity fluctuations it is useful to describe the fluctuations with two-point, two-time covariance statistics, i.e., the average product between a turbulence velocity component at a specified location on a given WECS blade at a specified time' with a turbulence velocity component at another specified location on a given WECS. blade at another specified time. If the locations, times, and velocity components are the same then

the covariance is the variance of the velocity component in question. If the velocity components in question are different we obtain a measure of the degree to which the velocity components are related, or correlated, at a fixed point on the WECS at a fixed time. If the locations and times are different we then obtain a measure of the degree to which the velocity components (same or different) are related between blade elements and between time points.

Consideration of the covariance statistics herein is limited to the homogeneous and isotropic case. Homogeneous turbulent flow corresponds to a flow in which all statistics (means, covariances, etc.) associated with velocity fluctuations at a set of arbitrary points in space and time do not vary upon spatial translation of the set of points keeping the relative positions of the points the same. Isotropic turbulence corresponds to the case in which the turbulence statistics do not vary upon imposing coordinate system rotations or coordinate axes reflections. Atmospheric boundary layer flow is vertically nonhomogeneous. Horizontal homogeneity depends on the degree of uniformity of the terrain roughness and heat 
transfer characteristics. The symmetry properties of atmospheric boundary layer turbulence depends upon the scale of the eddies. Thus, the relatively long wavelength or energy containing eddies, i.e., eddies with horizontal scale comparable to the integral scale of turbulence are anisotropic, while the relatively short wavelength eddies with horizontal scale comparable to or less than the height of the eddy above the surface of the earth tend to be isotropic. The homogeneous and isotropic case permits mathematical simplicity and at the same time retains much of the physical process we wish to describe. The homogeneous and isotropic turbulence model permits the calculation of the two-point, two-time covariance statistics following the WECS blade elements upon specification of the turbulence energy spectrum function in the sense of Batchelor (1960). In the nonhomogeneous and anisotropic case a single defining function, like the turbulence energy spectrum function, for calculation of all covariances following the WECS blade elements is not available. The turbulence energy spectrum function permits the calculation of the Eulerian covariance function matrix. The elements of the Eulerian covariance matrix are composed of the two-point, two-time velocity component covariances in a frame of reference attached to the earth. In lieu of the availability of a turbulence energy spectrum function in the nonhomogeneous and anisotropic case one must directly specify the components of the Eulerian covariance matrix to calculate the turbulence covariance statistics following the blade elements of a WECS.

The engineering motivation for determining turbulence covariance statistics following the WECS blade elements is three-fold. First, the turbulence covariance statistics can be used directly in linear response analyses to calculate the covariances of WECS responses. Second, the turbulence covariances can be used to simulate turbulence velocity fluctuations via band-limited white noise and shaping filters for linear and nonlinear WECS operations simulation studies. Finally, turbulence velocity covariances can be used to derive discrete gust imputs. All of the above inputs may be required in the WECS design process. Discrete inputs are typically used for preliminary design, while turbulence space-time series would be used in final design verification studies. Turbulence spectra and covariances are used typically during intermediate and final design studies. 
In the analysis that follows we derive the two-point, two-time turbulence statistics for WECS design via a technique first used by Etkin (1960) in the context of airplane dynamics. The starting point for the analysis is the definition of wind fields. We first specify the Eulerian wind field and partition it into a mean flow with a superimposed turbulent contribution. The coordinate system for this representation is at the WECS hub, but does not rotate with WECS blade elements. The Eulerian wind field is expanded via Taylor series expansion about the WECS hub. The Taylor series representation of the Eulerian field is evaluated at a specified blade element. We next proceed to calculate the turbulence velocity covariances following the WECS blade elements with this Taylor series representation of the turbulence field evaluated at the WECS blade elements. The resulting two-point, two-time covariance statistics calculated with the Taylor series expansion of the wind field about the hub results in a series with each term composed of a two-time statistic of the turbulence field, evaluated at the hub, multiplied by a function of the coordinates of the WECS blade elements in question. The analysis herein retains terms in the covariance series to second-order in space coordinates, which requires retention of terms to second-order in the Taylor series expansion of the wind field. Thus, the task of developing the two-point, two-time covariance statistics of turbulence velocity fluctuations for WECS design is reduced to determining the two-time statistics of the coefficients in the spatial Taylor series expansion of the Eulerian wind field about the WECS hub. To do this we assume that the turbulence is homogeneous and isotropic and that Taylor's hypothesis is valid. This permits the calculation of frequency domain spectra of the turbulence velocities and associated gradients at the WECS hub from a single defining function, namely the turbulence energy spectrum function (Batchelor, 1960). The spectra at the WECS hub can be used to calculate the two-time statistics of the coefficients in the spatial Taylor series expansion of the Eulerian field noted above. The Dryden turbulence model is used to calculate these frequency domain spectra. We next turn our attention to linear systems to discuss the application of the model developed herein. This leads to the development of nonstationary spectra of turbulence 
velocity fluctuations following the blade elements.

Appendices are provided which outline: 1) a procedure for the exact calculation of two-point, two-time velocity covariance functions following the WECS blade elements, and 2) a procedure for the calculation of the same covariance functions for the vertically nonhomogeneous atmospheric boundary layer using the concept of Taylor series expansion of the wind field about the hub. 


\section{WIND FIELDS}

In this section we calculate wind fluctuations following a WECS blade element. We shall use a Cartesian Coordinate system like that shown in Figure 1. The $x_{1}$ - and $x_{2}$-axes are parallel to the surface of the earth and the $x_{3}$-axis is directed along the vertical. The disk of rotation of the WECS lies in the $\left(x_{2}, x_{3}\right)$-plane with the hub at the origin of the coordinate system.

EULERIAN WIND FIELD

The $x_{i}$-component of the wind velocity vector is denoted by $u_{i}$. The quantity $u_{i}$ is a stochastic function of $x_{1}, x_{2}, x_{3}$, and $t$. The wind field is assumed to be statistically steady and horizontally homogeneous.

The ensemble average of the wind velocity vector is assumed to be given by

$$
\left\langle u_{i}\right\rangle=\bar{u}\left(x_{3}\right) \delta_{1 i}
$$

where

$$
\begin{aligned}
\langle\rangle & =\text { ensemble average } \\
\delta_{i j} & =\text { kronecker delta function } \\
\bar{u}\left(x_{3}\right) & =\text { mean wind profile. }
\end{aligned}
$$

The mean flow is directed along the $x_{1}$-axis and is a function of $x_{3}$ only, which is consistent with the assumption of statistical steadiness and horizontai homogeneity. The turbulence velocity fluctuations are obtained by subtracting the ensemble average velocity vector field from the wind field, i.e.,

$$
v_{i}^{\prime}=u_{i}-\left\langle u_{i}\right\rangle \text {. }
$$

The turbulence velocity fluctuations are stochastic functions of $x_{1}, x_{2}, x_{3}$, and $t$, and are statistically steady and fully homogeneous.

We expand the quantity $v_{j}^{\prime}$ about the origin of the coordinate system in the $\left(x_{2}, x_{3}\right)-p l a n e$, so that

$v_{i}^{\prime}\left(0, x_{2}, x_{3}, t\right)=w_{i}(t)+p_{i}(t) x_{2}+q_{j}(t) x_{3}+\frac{1}{2} P_{j}(t) x_{2}^{2}+\frac{1}{2} Q_{j}(t) x_{3}^{2}+M_{i}(t) x_{2} x_{3}$

where

$$
w_{j}(t)=v_{j}^{\prime}(0,0,0, t)
$$




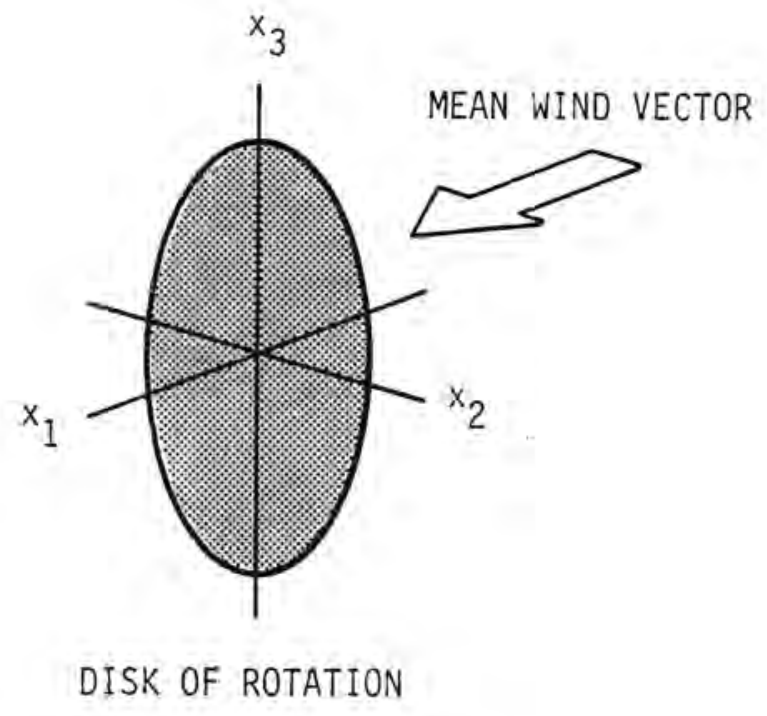

Figure 1. Frame of reference 


$$
\begin{aligned}
& p_{i}(t)=\left(\frac{\partial v_{i}^{\prime}}{\partial x_{2}}\right)_{0} \\
& q_{1}=\left(\frac{\partial v_{i}^{\prime}}{\partial x_{3}}\right)_{0} \\
& p_{i}(t)=\left(\frac{\partial^{2} v_{i}}{\partial x_{2}^{2}}\right)_{0} \\
& Q_{i}(t)=\left(\frac{\partial^{2} v_{i}^{-}}{\partial x_{3}^{2}}\right)_{0} \\
& M_{i}(t)=\left(\frac{\partial^{2} v_{j}}{\partial x_{2} \partial x^{\prime}}\right)_{0}
\end{aligned}
$$

and we have truncated the series to include second-order terms. The coefficients in the expansion are evaluated at the origin of the coordinate system and are functions of time only. The expansion of the Eulerian field given by Equation (3) is the basis for the analys is that follows.

\section{WIND FLUCTUATIONS $\underline{\text { AT }}$ A BLADE ELEMENT}

We define a reference line located in the WECS plane of rotation. The reference line (see Figure 2) is fixed with respect to the WECS blades. At time $t=0$ the reference 1 ine is coincident with the $x_{2}$-axis. At time $t \neq 0$ the angle between the reference line and the $x_{2}$-axis is $\omega_{0} t$, where $\omega_{0}$ is the angular rotation rate of the WECS. The angle between the reference 1 ine and the WECS blade line is $\alpha$. The trajectory coordinates of a point located at 


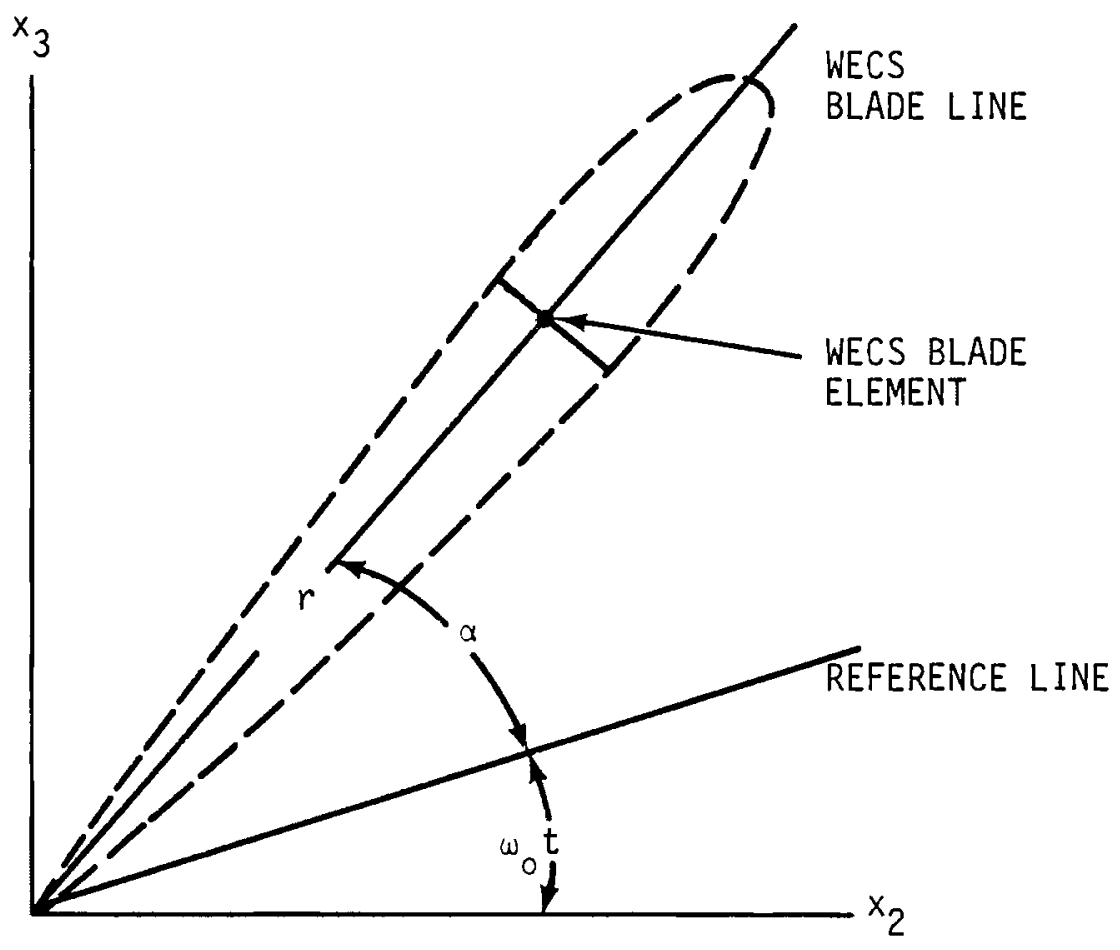

Figure 2. WECS blade coordinate system 
radial distance $r$ on the blade line are given by

$$
\begin{gathered}
x_{1}(t)=0 \\
x_{2}(t)=r \cos \left(\omega_{0} t+\alpha\right) \\
x_{3}(t)=r \sin \left(\omega_{0} t+\alpha\right) .
\end{gathered}
$$

The angle a serves to define the angular location of a WECS blade and radial distance $r$ serves to locate a blade element along the blade line. The number of values that a can take on is equal to the number of blades. Evaluation of the Eulerian wind field at a blade element located at radial distance $r$ along the blade line which in turn is located at angular distance a from the WECS reference line is given by

$$
v_{i}^{\prime}\left(x_{1}(t), x_{2}(t), x_{3}(t), t\right)=v_{i}(t ; r, \alpha)
$$

where

$$
\begin{aligned}
v_{i}(t ; r, \alpha) & =w_{i}(t)+p_{i}(t) r \cos \left(\omega_{0} t+\alpha\right) \\
& +q_{j}(t) r \sin \left(\omega_{0} t+\alpha\right)+\frac{1}{2} P_{i}(t) r^{2} \cos ^{2}\left(\omega_{0} t+\alpha\right) \\
& +\frac{1}{2} Q_{j}(t) r^{2} \sin ^{2}\left(\omega_{0} t+\alpha\right) \\
& +M_{j}(t) r^{2} \cos \left(\omega_{0} t+\alpha\right) \sin \left(\omega_{0} t+\alpha\right)
\end{aligned}
$$

Equation (14) permits the calculation of turbulence velocity fluctuations at the blade element in question upon specification of the wind processes $w_{j}(t)$, $p_{j}(t), q_{j}(t), P_{j}(t) Q_{j}(t)$, and $M_{j}(t)$ at the WECS hub and the rotation of the blade element $\omega_{0}$ and position $r, \alpha$. It should be kept in mind that the turbulence velocity fluctuations in Equation (14) are velocities, following a WECS blade element, in a frame of reference fixed with respect to the ground. 


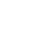




\section{COVARIANCES}

In this section we calculate the covariance matrix of the turbulent velocity fluctuations following the WECS blade elements. We consider two blade elements with coordinates $\left(r_{1}, \alpha_{1}\right)$ and $\left(r_{2}, \alpha_{2}\right)$ with respect to the WECS reference line as given in Figure 3. Multiplication of $v_{i}$ evaluated at time $t+\tau$ at blade element located at $\left(r_{1}, \alpha_{1}\right)$ with $v_{j}$ evaluated at time $t$ at blade element located at $\left(r_{2}, \alpha_{2}\right)$, ensemble averaging the resulting relationship, and retention of terms to second-order in $r$ yields

$$
R_{v_{i} v_{j}}\left(\tau ; t, r_{1}, r_{2}, \alpha_{1}, \alpha_{2}\right)=S_{i j}\left(\tau ; r_{1}, r_{2}, \alpha_{1}, \alpha_{2}\right)+N_{i j}\left(\tau ; t, r_{1}, r_{2}, \alpha_{1}, \alpha_{2}\right)
$$

where

$$
\begin{aligned}
& S_{i j}\left(\tau ; r_{1}, r_{2}, \alpha_{1}, \alpha_{2}\right)=R_{w_{i} w_{j}}(\tau)+\frac{r_{2}^{2}}{2}\left[R_{w_{i} P_{j}}(\tau)+R_{w_{i} Q_{j}}(\tau)\right] \\
& +\frac{r_{1}^{2}}{2}\left[R_{P_{i} w_{j}}(\tau)+R_{Q_{j} w_{j}}(\tau)\right]+\frac{r_{1} r_{2}}{2}\left[R_{p_{i} p_{j}}(\tau)+R_{q_{j} q_{j}}(\tau)\right] \\
& \cos \left(\omega_{0} \tau+\alpha_{1}-\alpha_{2}\right)+\frac{r_{1} r_{2}}{2}\left[R_{q_{j} p_{j}}(\tau)-R_{p_{j} q_{j}}(\tau)\right] \cdot \\
& \sin \left(\omega_{0} \tau+\alpha_{1}-\alpha_{2}\right)
\end{aligned}
$$




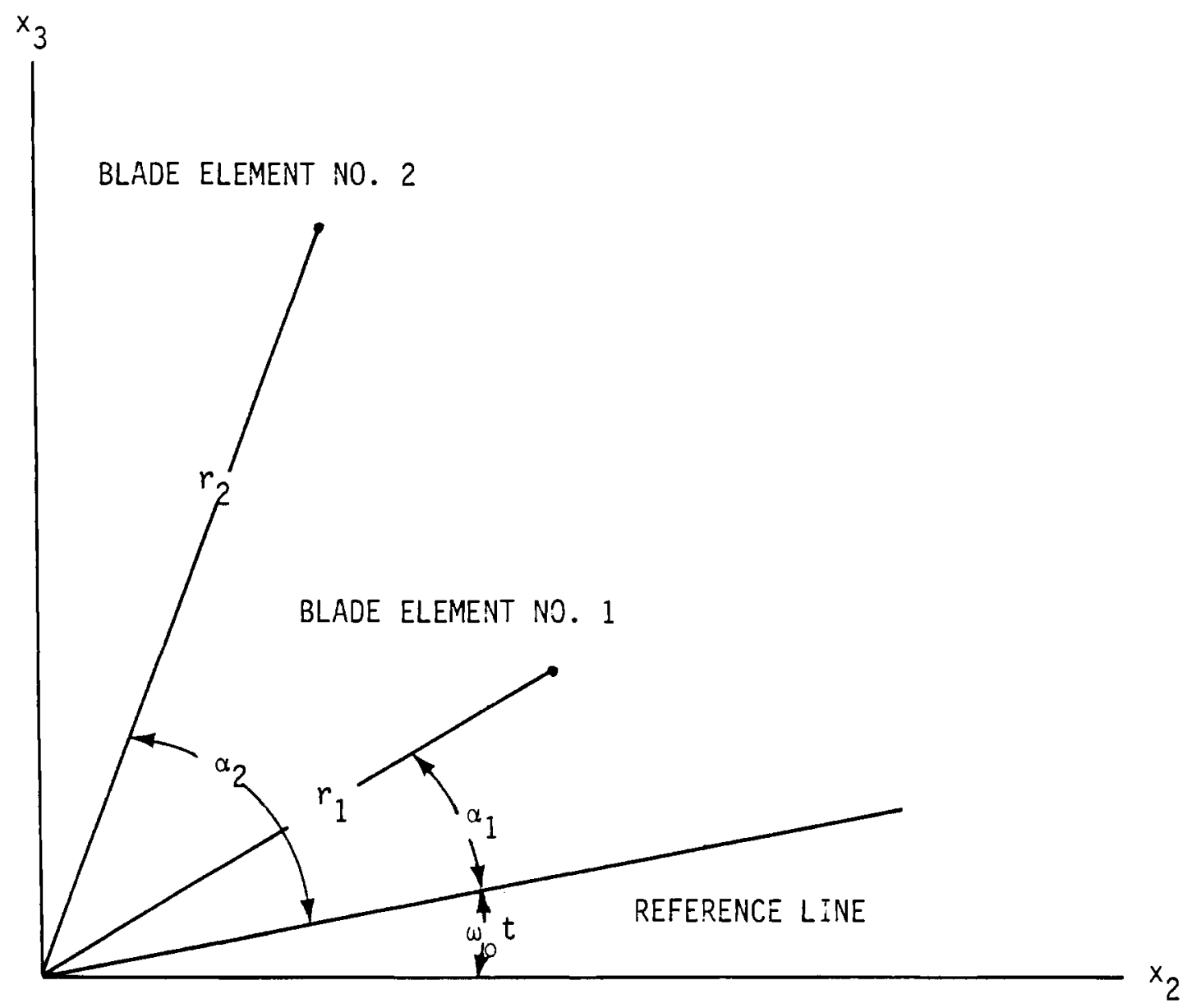

Figure 3. Two-point WECS blade geometry 
and

$$
\begin{aligned}
& N_{i j}\left(\tau ; t, r_{1}, r_{2}, \alpha_{1}, \alpha_{2}\right)=r_{2} R_{w_{j} p_{j}}(\tau) \cos \left(\omega_{0} t+\alpha_{2}\right) \\
& +r_{2} R_{w_{j}} q_{j}(\tau) \sin \left(\omega_{0} t+\alpha_{2}\right) \\
& +r_{1} R_{p_{j} w_{j}}(\tau) \cos \left(\omega_{0} t+\omega_{0} \tau+\alpha_{1}\right) \\
& +r_{1} R_{q_{j} w_{j}}(\tau) \sin \left(\omega_{0} t+\omega_{i} \bar{\zeta}+\alpha_{1}\right) \\
& +\frac{r_{1} r_{2}}{2}\left[R_{p_{j} p_{j}}(\tau)-R_{q_{j} q_{j}}(\tau)\right] \cos \left(2 \omega_{0} t+\omega_{0} \tau+\alpha_{1}+\alpha_{2}\right) \\
& +\frac{r_{1} r_{2}}{2}\left[R_{p_{i} q_{j}}(\tau)+R_{q_{j} p_{j}}(\tau)\right] \sin \left(2 \omega_{0} t+\omega_{0} \tau+\alpha_{1}+\alpha_{2}\right) \\
& \begin{aligned}
+\frac{r_{2}^{2}}{4} & {\left[R_{w_{i} P_{j}}(\tau)-R_{w_{i} Q_{j}}(\tau)\right] \cos \left(2 \omega_{0} t+2 \alpha_{2}\right) } \\
+\frac{r_{1}^{2}}{4} & {\left[R_{P_{i} w_{j}}(\tau)-R_{Q_{i} w_{j}}(\tau)\right] \cos \left(2 \omega_{0} t+2 \omega_{0} \tau+2 \alpha_{1}\right) }
\end{aligned} \\
& +\frac{r_{2}^{2}}{2} R_{w_{i} M_{j}}(\tau) \sin \left(2 \omega_{0} t+2 \alpha_{2}\right) \\
& +\frac{r_{1}^{2}}{2} R_{M_{j} w_{j}}(\tau) \sin \left(2 \omega_{0} t+2 \omega_{0} \tau+2 \alpha_{1}\right)
\end{aligned}
$$

with

$$
R_{\xi_{j} \zeta_{j}}(\tau)=\left\langle\xi_{j}(t+\tau) \zeta_{j}(t)\right\rangle
$$

The quantities $S_{i j}$ and $N_{i j}$ represent the stationary and nonstationary contributions to the temporal gust covariance with respect to the blade elements. Both the stationary and nonstationary contributions are constructed from the temporal covariance functions of the stationary processes $w, p, q, P, Q$, and $M$ at the hub. The results given by Equations $(15,16,17)$ apply to inhomogeneous and anisotropic turbulent flow. 

The three-fold wave number-frequency decomposition of $v_{i}\left(x_{1}, x_{2}, x_{3}, t\right)$ is given by

$$
v_{i}^{\prime}\left(x_{1}, x_{2}, x_{3}, t\right)=\int_{-\infty}^{\infty} e^{i\left(x_{j} k_{j}+\omega t\right)} d z_{i}\left(k_{1}, k_{2}, k_{3}, \omega\right)
$$

where

$$
\begin{aligned}
k_{j} & =\text { wavenumber along } x_{j} \text {-axis } \\
w & =\text { radian frequency } \\
d z_{i} & =\text { Fourier amplitude }
\end{aligned}
$$

$\int_{-\infty}^{\infty}() d z_{i}=$ fourfold stochastic Fourier integral over wavenumber and frequency. (4)

$$
i=\sqrt{-1} \quad \text {. }
$$

The Einstein summation convention is applicable.

We define

$$
\xi_{i k m v \lambda}(t)=\left(\frac{\partial v+\lambda v_{i}}{\partial x_{k}^{v} \partial x_{m}^{\lambda}}\right)_{0}
$$

where

$$
()_{0}=\text { evalution at WECS hub. }
$$

The Einstein summation convention does not apply to Greek indices. By appropriate selection of the subscripts on $\xi$ we can obtain any of the coefficients in the Taylor series expansion given by Equation (3). Substitution of Equation (19) into Equation (20) yields

$$
\xi_{i k m \nu \lambda}(t)=\int_{-\infty}^{\infty} e^{i \omega t}\left(i_{k_{k}}\right)^{\nu}\left(i_{k_{m}}\right)^{\lambda} d z_{i}\left(\kappa_{1}, k_{2}, k_{3}, \omega\right) .
$$

(4) 
COVARIANCES AND SPECTRA

We construct the following covariance function

$$
\begin{aligned}
R_{\xi_{i k m u \lambda} \xi_{j s n_{B \gamma}}(\tau)}=\int_{\substack{(4) \\
(4)}}^{\infty}\left(i_{k_{k}}\right)^{\nu}\left(i_{k_{m}}\right)^{\lambda}\left(-i_{k_{s}}\right)^{\beta}\left(-i_{k_{n}}\right)^{\gamma} e^{i \omega \tau} . \\
\phi_{i j}\left(k_{1}, k_{2}, k_{3}, \omega\right) d_{k_{1}} d_{k_{2}} d_{k_{3}} d \omega
\end{aligned}
$$

where

$$
\begin{aligned}
\phi_{i j}\left(k_{1}, k_{2}, k_{3}, w\right)= & \text { three-fold wave number-frequency } \\
& \text { spectral density function of } \\
& v_{i}^{\prime}\left(x_{1}, x_{2}, x_{3}, t\right) \text { and } v_{j}^{\prime}\left(x_{1}, x_{2}, x_{3}, t\right) .
\end{aligned}
$$

Thus, upon specification of $\phi_{i j}\left(k_{1}, k_{2}, k_{3}, w\right)$ all of the relevant covariance functions in $S_{i j}$ and $N_{i j}$ and hence, in $R_{v_{i}} v_{j}$ can be determined.

We assume Taylor's hypothesis is valid, so that

$$
\omega=k_{1} u_{0}
$$

and

$$
\dot{\phi}_{i j}\left(k_{1}, k_{2}, k_{3}, w\right)=\phi_{i j}\left(k_{1}, k_{2}, k_{3}\right) \delta\left(k_{1} u_{0}-w\right)
$$

where

$$
\delta\left(\kappa_{1} u_{0}-\omega\right)=\text { Dirac delta function } \text {. }
$$

This assumes

$$
\bar{u}\left(x_{3}\right)=\bar{u}(0)=u_{0}
$$

i.e., the wind profile is assumed to be constant. Substitution of Equation (23) into Equation (22) yields

$$
R_{\xi_{i k m \nu \lambda} \xi_{j s n B \gamma}}(\tau)=\int_{-\infty}^{\infty} \xi_{i k m \cup \lambda} \xi_{j s n B \gamma}(\omega) e^{i \omega \tau} d \omega
$$


where

$$
\Psi_{\xi_{i k m \nu \lambda} \xi_{j s n \beta \gamma}}(\omega)=\int_{-\infty}^{\infty}\left(i \kappa_{k}\right)^{\nu}\left(i \kappa_{m}\right)^{\lambda}\left(-i \kappa_{s}\right)^{\beta}\left(-i \kappa_{n}\right)^{\gamma} .
$$

$$
\frac{1}{u_{0}} \phi_{i j}\left(\frac{\omega}{u_{0}}, k_{2}, k_{3}\right) d_{k_{2}} d_{k_{3}}
$$

According to Batchelor (1960)

$$
\phi_{i j}\left(k_{1}, k_{2}, k_{3}\right)=\frac{E(k)}{4 \pi \kappa^{4}}\left(k^{2} \delta_{i j}-\kappa_{i} k_{j}\right)
$$

where

$$
\kappa=\left(\kappa_{1}^{2}+\kappa_{2}^{2}+\kappa_{3}^{2}\right)^{\frac{1}{2}}
$$

and $E(k)$ is the energy spectrum function. As stated earlier by proper selection of the subscripts on $\Psi$ in Equation (26) we can generate the frequency spectral density function for any coefficient in Equation (14). Thus, if we set $\nu=\lambda=0, \beta=0, \gamma=1, n=2$ then Equation (26) yields the crossspectral density function for $w_{j}(t)$ and $q_{j}(t)$, namely

$$
\Psi_{w_{i} q_{j}}(\omega)=\int_{-\infty}^{\infty}-\frac{i \kappa_{3}}{n_{0}} \phi_{i j}\left(\frac{\omega}{u_{0}}, \kappa_{2}, k_{3}\right) d k_{2} d_{k_{3}} .
$$

It can be shown that

$$
\begin{gathered}
\Psi_{w_{i} p_{j}}(\omega)=-\Psi_{p_{i} w_{j}}(\omega) \\
\Psi_{w_{j} q_{j}}(\omega)=-\Psi_{q_{j} w_{j}}(\omega) \\
\Psi_{w_{i} p_{j}}(\omega)=\Psi_{p_{i} w_{j}}(\omega)=-\Psi_{p_{i} p_{j}}(\omega)
\end{gathered}
$$




$$
\begin{gathered}
\Psi_{w_{i} Q_{j}}(\omega)=\Psi_{Q_{j} w_{j}}(\omega)=-\Psi_{q_{j} q_{j}}(\omega) \\
\Psi_{w_{i} M_{j}}(\omega)=\Psi_{M_{j} w_{j}}(\omega)=-\Psi_{q_{j} p_{j}}(\omega)=-\Psi_{p_{i} q_{j}}(\omega)
\end{gathered}
$$

with similar relationships for corresponding covariances. Thus,

$$
\begin{gathered}
s_{i j}\left(\tau ; r_{1}, r_{2}, \alpha_{1}, \alpha_{2}\right)=R_{w_{j} w_{j}}(\tau)-\left[R_{p_{j} p_{j}}(\tau)+R_{q_{j} q_{j}}(\tau)\right] \\
\frac{1}{2}\left[r_{1}^{2}+r_{2}^{2}-r_{1} r_{2} \cos \left(\omega_{0} \tau+\alpha_{1}-\alpha_{2}\right)\right] \\
N_{i j}\left(\tau ; t, r_{1}, r_{2}, \alpha_{1}, \alpha_{2}\right)=R_{w_{j} p_{j}}(\tau)\left[r_{2} \cos \left(\omega_{0} t+\alpha_{2}\right)-\right. \\
\left.r_{1} \cos \left(\omega_{0} t+\omega_{0} \tau+\alpha_{1}\right)\right]+R_{w_{j} q_{j}}(\tau)\left[r_{2} \sin \left(\omega_{0} t+\alpha_{2}\right)-\right. \\
\left.r_{1} \sin \left(\omega_{0} t+\omega_{0} \tau+\alpha_{1}\right)\right]-\frac{1}{2} R_{q_{i} p_{j}}(\tau)\left[r_{1}^{2} \sin \left(2 \omega_{0} t+2 \omega_{0} \tau+2 \alpha_{1}\right)\right. \\
\left.+r_{2}^{2} \sin \left(2 \omega_{0} \tau+2 \alpha_{2}\right)-2 r_{1} r_{2} \sin \left(2 \omega_{0} t+\omega_{0} \tau+\alpha_{1}+\alpha_{2}\right)\right] \\
+\frac{1}{4}\left[R_{q_{j} q_{j}}(\tau)-R_{p_{j} p_{j}}(\tau)\right]\left[r_{1}^{2} \cos \left(2 \omega_{0} t+2 \omega_{0} \tau+2 \alpha_{1}\right)\right. \\
\left.+r_{2}^{2} \cos \left(2 \omega_{0} t+2 \alpha_{2}\right)-2 r_{1} r_{2} \cos \left(2 \omega_{0} t+\omega_{0} \tau+\alpha_{1}+\alpha_{2}\right)\right]
\end{gathered}
$$

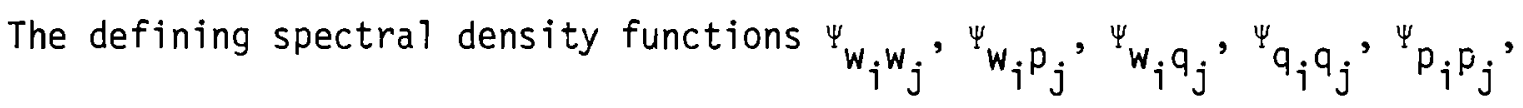
and ${ }^{\Psi} q_{j} p_{j}$ can be Fourier transformed according to Equation (25) to yield the relevant covariance functions in Equations (35) and (36). These spectral dencity functions in terms of the energy function, $E(k)$, are as follows

$$
\Psi_{w_{1} w_{1}}(\omega)=\frac{1}{4 \pi u_{0}} \int_{-\infty}^{\infty} \frac{E^{(2)}\left(k_{0}\right)}{k_{0}^{4}}\left(k_{2}^{2}+k_{3}^{2}\right) d k_{2} d k_{3}
$$

18 


$$
\begin{aligned}
& \Psi_{w_{2} w_{2}}(\omega)=\frac{1}{4 \pi u_{0}} \int_{\substack{(2) \\
(2)}}^{\infty} \frac{E\left(k_{0}\right)}{k_{0}^{4}}\left(\frac{\omega^{2}}{u_{0}^{2}}+k_{3}^{2}\right) d_{k_{2}} d k_{3} \\
& \Psi_{w_{3} w_{3}}(\omega)=\Psi_{w_{2} w_{2}}(\omega) \\
& \psi_{w_{j} w_{j}}(\omega)=0, i \neq j \\
& \Psi_{w_{j} p_{j}}(\omega)=-\left(\delta_{i 1} \delta_{j 2}+\delta_{i 2} \delta_{j 1}\right) \frac{i \omega}{4 \pi u_{0}^{2}} \int_{-\infty}^{\infty} \frac{k_{2}^{2} E\left(k_{0}\right)}{k_{0}^{4}} d k_{2} d k_{3} \\
& \psi_{w_{j} q_{j}}(\omega)=-\left(\delta_{i 1} \delta_{j 3}+\delta_{j 3} \delta_{j 1}\right) \frac{i \omega}{4 \pi u_{0}^{2}} \int_{-\infty}^{\infty} \frac{\kappa_{3}^{2} E\left(\kappa_{0}\right)}{k_{0}^{4}} d k_{2} d k_{3} \\
& \Psi_{p_{1} p_{1}}(\omega)=\frac{1}{4 \pi u_{0}} \int_{\substack{-\infty \\
(2)}}^{\infty} \frac{\kappa_{2}^{2} E\left(\kappa_{0}\right)}{\kappa_{0}^{4}}\left(\kappa_{2}^{2}+\kappa_{3}^{2}\right) d k_{2} d k_{3} \\
& \Psi_{p_{2} p_{2}}(\omega)=\frac{1}{4 \pi u_{0}} \int_{-\infty}^{\infty} \frac{\kappa_{2}^{2} E\left(k_{0}\right)}{k_{0}^{4}}\left(\frac{\omega^{2}}{u_{0}^{2}}+k_{3}^{2}\right) d k_{2} d k_{3} \\
& \Psi_{p_{3} p_{3}}(\omega)=\frac{1}{4 \pi u_{0}} \int_{-\infty}^{\infty} \frac{\kappa_{2}^{2} E\left(\kappa_{0}\right)}{k_{0}^{4}}\left(\frac{\omega^{2}}{u_{0}^{2}}+\kappa_{2}^{2}\right) d k_{2} d k_{3} \\
& \Psi_{p_{j} p_{j}}(\omega)=0, j \neq j
\end{aligned}
$$




$$
\begin{aligned}
& \Psi q_{1} q_{1}(\omega)=\frac{1}{4 \pi i_{0}} \int_{\substack{-\infty \\
(2)}}^{\infty} \frac{\kappa^{2} E\left(\kappa_{0}\right)}{\kappa_{0}^{4}}\left(\kappa_{2}^{2}+\kappa_{3}^{2}\right) d_{k} d_{k_{3}}=\Psi_{p_{1} p_{1}}(\omega) \\
& \Psi_{q_{2} q_{2}}(\omega)=\frac{1}{4 \pi u_{0}} \int_{\substack{-\infty \\
(2)}}^{\infty} \frac{\kappa_{3}^{2} E\left(\kappa_{0}\right)}{\kappa_{0}^{4}}\left(\frac{\omega^{2}}{u_{0}^{2}}+\kappa_{3}^{2}\right) d_{k_{2}} d_{\kappa_{3}}=\Psi_{p_{3} p_{3}}(\omega) \\
& \Psi q_{3} q_{3}(\omega)=\frac{1}{4 \pi u} \int_{\substack{-\infty \\
(2)}}^{\infty} \frac{\kappa_{3}^{2} E\left(\kappa_{0}\right)}{\kappa_{0}^{4}}\left(-\frac{\omega^{2}}{u_{0}^{2}}+\kappa_{2}^{2}\right) d k_{2} d k_{3}=\Psi_{p_{2} p_{2}}(\omega) \\
& { }_{q_{j} q_{j}}(\omega)=0, \quad i \neq j \\
& \Psi_{q_{j} p_{j}}(\omega)=-\frac{\left(\delta_{2 j} \delta_{3 j}+\delta_{3 i} \delta_{2 j}\right)}{4 \pi u_{0}} \quad \int_{-\infty}^{\infty} \frac{\kappa_{2}^{2} \kappa_{3}^{2}}{\kappa_{0}^{4}} E\left(\kappa_{0}\right) d k_{2} d \kappa_{3}
\end{aligned}
$$

with

$$
\kappa_{0}=\left(\frac{\omega^{2}}{u_{0}^{2}}+\kappa_{2}^{2}+\kappa_{3}^{2}\right)^{\frac{1}{2}} \text {. }
$$

If $\omega_{0}=0$ the quantity $N_{i j}$ does not depend on time, so that the source of the nonstationary contribution to the turbulence covariance matrix following the WECS blade elements is the variation in time of the orientation of the line which connects the points in the two-point, two-time turbulence covariance matrix. Thus, consider the two points $A$ and $B$ on the $x_{2}$-axis in Figure 4.a. At $\tau=0$ the statistics $\left\langle\left(v_{2}\right)_{A}\left(v_{2}\right)_{B}\right\rangle$ and $\left\langle\left(v_{3}\right)_{A}\left(v_{3}\right)_{B}\right\rangle$ are 1ongitudinal and lateral covariances, respectively. After a clockwise quarter turn the points $A$ and $B$ are located along the $x_{3}$-axis as shown in Figure 4.b. At $\tau=0$ the statistics $\left\langle\left(v_{2}\right)_{A}\left(v_{2}\right)_{B}\right\rangle$ and $\left\langle\left(v_{3}\right)_{A}\left(v_{3}\right)_{B}\right\rangle$ are now lateral and longitudinal covariances, respectively. However, at $\tau=0$ the covariance $\left\langle\left(v_{1}\right)_{A}\left(v_{1}\right)_{B}\right\rangle$ does not vary with time because $v_{1}$ is normal to the WECS plane of rotation. For $\tau \neq 0$, all components in the covariance matrix $R_{v_{i} v_{j}}$ vary with time $t$ and, except for 


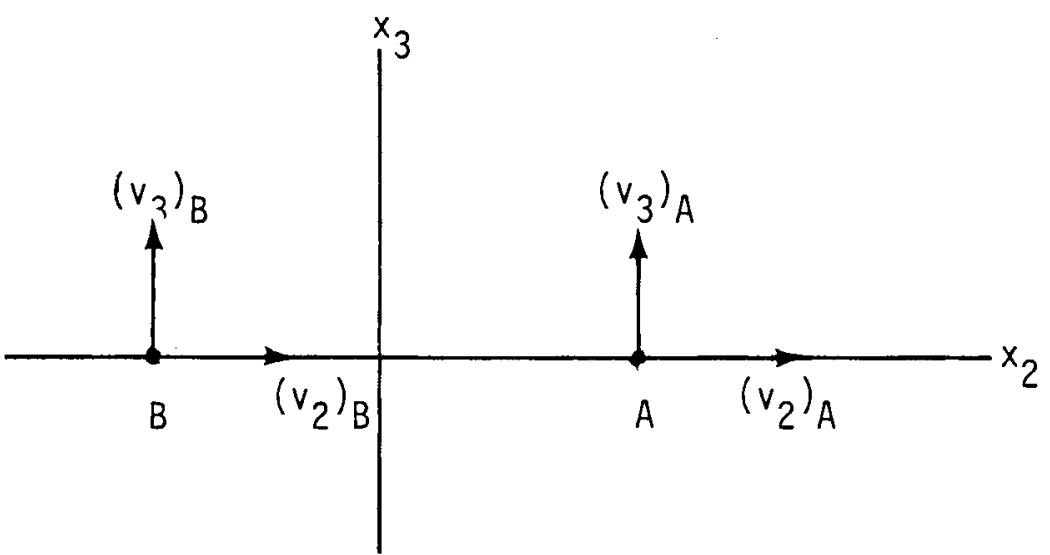

(a)

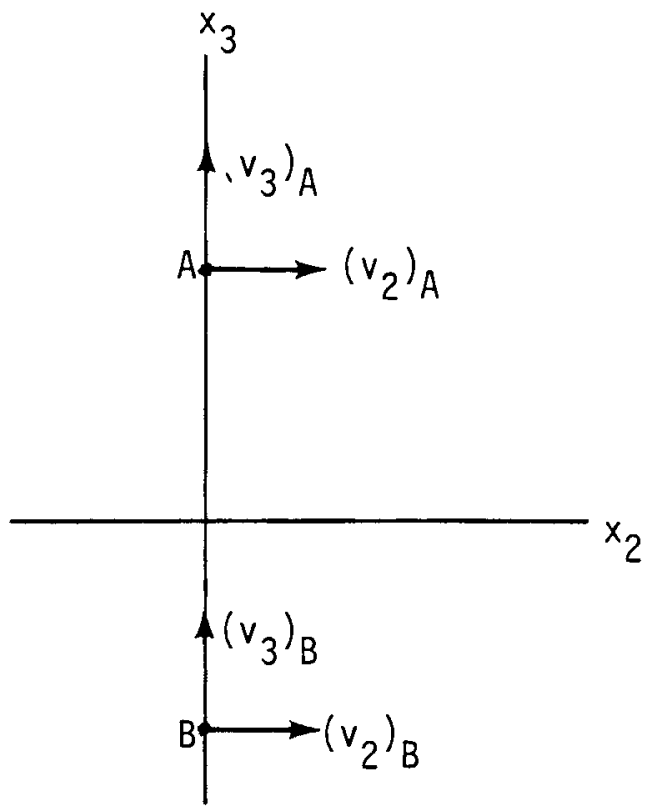

(b)

Figure 4. Longitudinal and lateral covariances at $\tau=0$ 
selected values of $t$ and $\tau$, are neither longitudinal nor lateral covariances.

Appendix A provides a discussion of the exact calculation of the two-point, two-point covariance statistics for isotropic and homogeneous turbulence.

Appendix $B$ provides a discussion of the calculation of the two-point, two-time covariance statistics for the nonhomogeneous case using the formalism developed in the previous section and a generalization of the procedures developed in this section.

\section{A TRANSFORMATION}

It may be useful to reference the components of the turbulence velocity vector to a frame of reference $\left(x_{1}^{\prime}, x_{2}^{\prime}, x_{3}^{\prime}\right)$ which rotates with the WECS blade elements, such that the $x_{1}^{\prime}$-axis is parallel to the $x_{1}$-axis, the $x_{2}$-axis is parallel to the WECS blade line in Figure 5 and the $x_{3}$-axis lies in the plane of rotation and is normal to the WECS blade line. This coordinate system has the advantage that at $\tau_{,}=0$ the covariances $\left\langle\left(v_{2}^{\prime}\right)_{A}\left(v_{2}^{\prime}\right)_{B}\right\rangle$ and $\left\langle\left(v_{3}^{\prime}\right)_{A}\left(v_{3}^{\prime}\right)_{B}\right\rangle$ are time invariant, where $v_{i}$ denotes the component of velocity along the $x_{i}^{\prime}$-axis. The relationship between $v_{i}^{\prime}$ and $v_{k}$ is

$$
v_{i}^{\prime}=T_{i k} v_{k}
$$

where the transformation matrix $T_{i k}$ is given by

$$
T_{i k}=\left\{\begin{array}{ccc}
1 & 0 & 0 \\
0 & \cos \left(\omega_{0} t+\alpha\right) & \sin \left(\omega_{0} t+\alpha\right) \\
0 & \sin \left(\omega_{0} t+\alpha\right) & -\cos \left(\omega_{0} t+\alpha\right)
\end{array}\right\} .
$$

The covariances in the primed frame are related to the ones in the unprimed frame through the following relationship

$$
R_{v_{i} v_{j}^{\prime}}(\tau ; t)=T_{i k}(t+\tau) T_{j m}(t) R_{v_{k} v_{m}}(\tau ; t)
$$

\section{ENERGY SPECTRUM FUNCTIONS}

The most widely used functions for $E(k)$ are those that correspond to the von Karman and Dryden turbulence models (Lumley and Panofsky, 1964, and Etkin, 


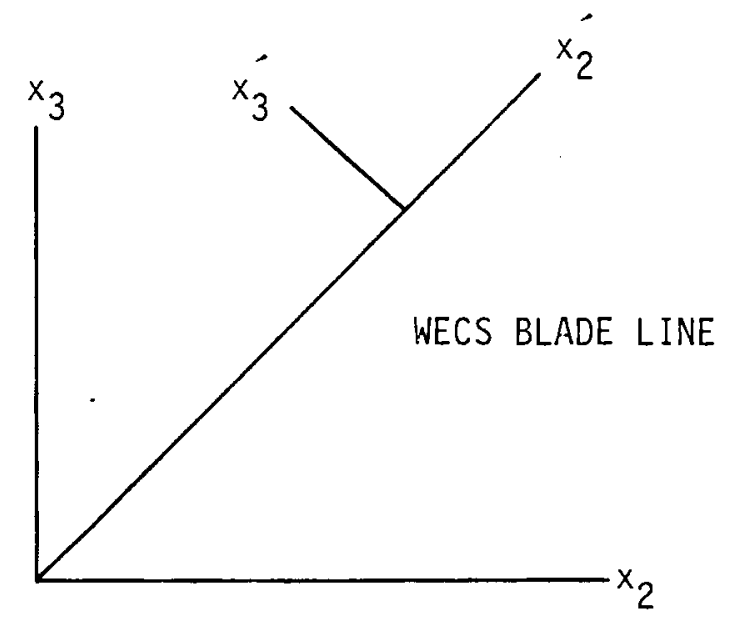

Figure 5. Relationship between coordinates (prime) following blade element and stationary coordinates (unprimed) 
1972), namely,

von Karman:

$$
E(\kappa)=\frac{55}{9} \frac{\sigma^{2} L}{\pi} \frac{(1.339 L K)^{4}}{\left[1+(1.339 L K)^{2}\right]^{17 / 6}}
$$

Dryden:

$$
E(x)=\frac{8 \sigma^{2} L}{\pi} \frac{\left(L_{K}\right)^{4}}{\left[1+\left(L_{K}\right)^{2}\right]^{3}}
$$

where $L$ and $\sigma$ denote the integral scale and standard deviation of turbulence. CALCULATION OF SPECTRAL FUNCTIONS

Substitution of these functions into Equations (37) through (51) yields the auto- and cross-spectral density functions of $w, p$, and $q$. We shall use the Dryden model because it leads to closed form algebraic expressions for these spectra. Because of the finite extent of the WECS the integrals in Equations (37) through (51) must be truncated so as not to include Fourier components with wave numbers $k_{2}, k_{3}>2 \pi / b$ which are inconsistent with the truncated Taylor series approximation, Equation (3). The quantity $b$ is a characteristic length such that upon removing Fourier components with $\kappa_{2}, k_{3}>2 \pi / b$ from the wind field a turbulence process which satisfies the truncated Taylor series approximation Equation (3) is obtained, i.e., the third-and higher-order terms are negligibly small. Later we shall obtain an estimate of the quantity b when we examine the convergence properties of the Taylor series representation of the turbulence velocity field.

Substitution of the energy spectrum function for the Dryden model into Equation (37) through (51) yields

$$
\frac{2 \omega \Psi w_{1} w_{1}(\omega)}{\sigma^{2}}=G(\Omega)=\frac{2}{\pi}\left\{\frac{1}{1+\Omega^{2}}-\frac{1+\Omega^{2}+2 \Omega_{c}^{2}}{\left(1+\Omega^{2}+\Omega_{c}^{2}\right)^{2}}\right\} \Omega
$$




$$
\begin{aligned}
& \frac{{ }^{2 \omega \Psi} w_{2} W_{2}(\omega)}{\sigma^{2}}=\frac{2 \omega{ }^{2} w_{3} W_{3}(\omega)}{\sigma^{2}}=\frac{1}{\pi}\left\{\frac{1+3 \Omega^{2}}{\left(1+\Omega^{2}\right)^{2}}-\frac{1+3 \Omega^{2}+2 \Omega_{c}^{2}}{\left(1+\Omega^{2}+\Omega_{c}^{2}\right)^{2}}\right\} . \Omega \\
& \frac{{ }^{2 \omega \Psi_{w} w_{j}}(\omega)}{\sigma^{2}}=0, i \neq j \\
& \frac{{ }^{2 L \omega \Psi} w_{j} p_{j}(\omega)}{\sigma^{2}}=-\left(\delta_{i 1} \delta_{j 2}+\delta_{i 2} \delta_{j 1}\right) i \frac{\Omega G(\Omega)}{2} \\
& \frac{{ }^{2 L \omega \Psi} w_{j} q_{j}(\omega)}{\sigma^{2}}=-\left(\delta_{i 1} \delta_{j 3}+\delta_{i 3} \delta_{j 1}\right) i \frac{\Omega G(\Omega)}{2}
\end{aligned}
$$

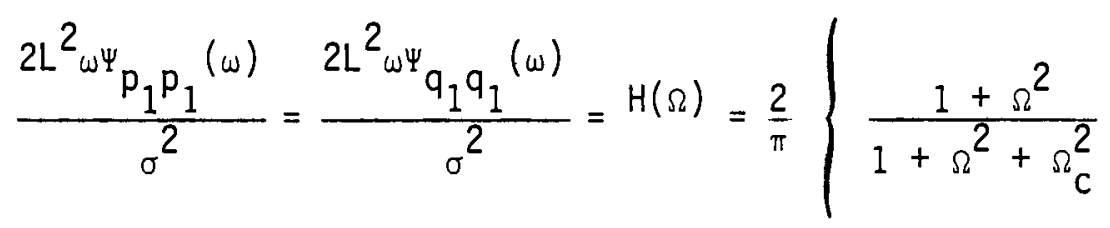

$$
\begin{aligned}
& \left.+\log \left(\frac{1+\Omega^{2}+\Omega_{c}^{2}}{1+\Omega^{2}}\right)-\frac{1}{2} \frac{\Omega_{c}^{4}}{\left(1+\Omega^{2}+\Omega_{c}^{2}\right)^{2}}-1\right) \Omega
\end{aligned}
$$

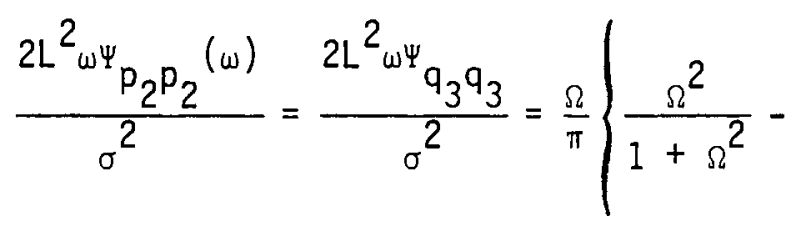

$$
\begin{aligned}
& \left.\frac{\Omega^{2}\left(1+\Omega^{2}+2 \Omega_{c}^{2}\right)}{\left(1+\Omega^{2}+\Omega_{c}^{2}\right)^{2}}\right\}+\frac{1}{4} H(\Omega)
\end{aligned}
$$




$$
\begin{aligned}
& \frac{2 L^{2} \omega{ }^{2} p_{3} p_{3}(\omega)}{\sigma^{2}}=\frac{2 L^{2}{ }^{2} q_{2} q_{2}(\omega)}{\sigma^{2}} \\
& =\frac{\Omega}{\pi}\left\{\frac{\Omega^{2}}{1+\Omega^{2}}-\frac{\Omega^{2}\left(1+\Omega^{2}+2 \Omega_{c}^{2}\right)}{\left(1+\Omega^{2}+\Omega_{c}^{2}\right)^{2}}\right\}+\frac{3}{4} \cdot H(\Omega)
\end{aligned}
$$

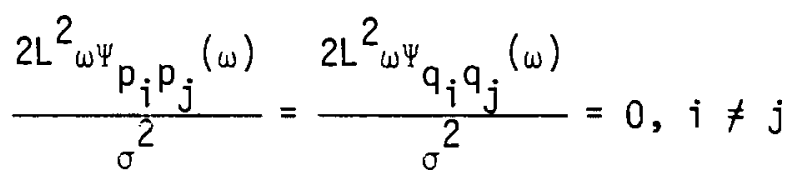

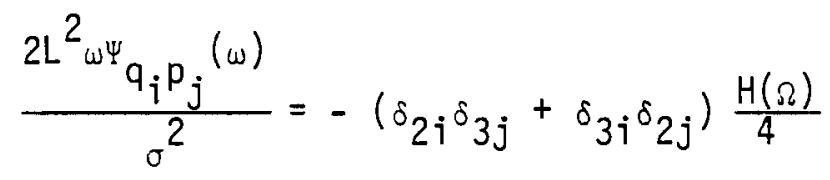

where

$$
\begin{aligned}
& \Omega_{0}=\frac{\omega L}{u_{0}} \\
& \Omega_{C}=\frac{2 \pi L}{b} .
\end{aligned}
$$

These spectra are sufficient for calculation of the second moment statistics of turbulence velocity fluctuations, following the WECS blade elements, for the truncated Taylor series expansion Equation (3) of the velocity field. Later we shall discuss the nonstationary spectra of the gust velocity components following the blade elements.

Figures 6 through 10 provides plots of the non-zero functions contained in Equations (58 through 67). The range of the parameter $\Omega_{C}$ is sufficiently wide to encompass the full range of WECS'S anticipated to be constructed in the future, i.e., diameters ranging between $10 \mathrm{~m}$ to $300 \mathrm{~m}$. 


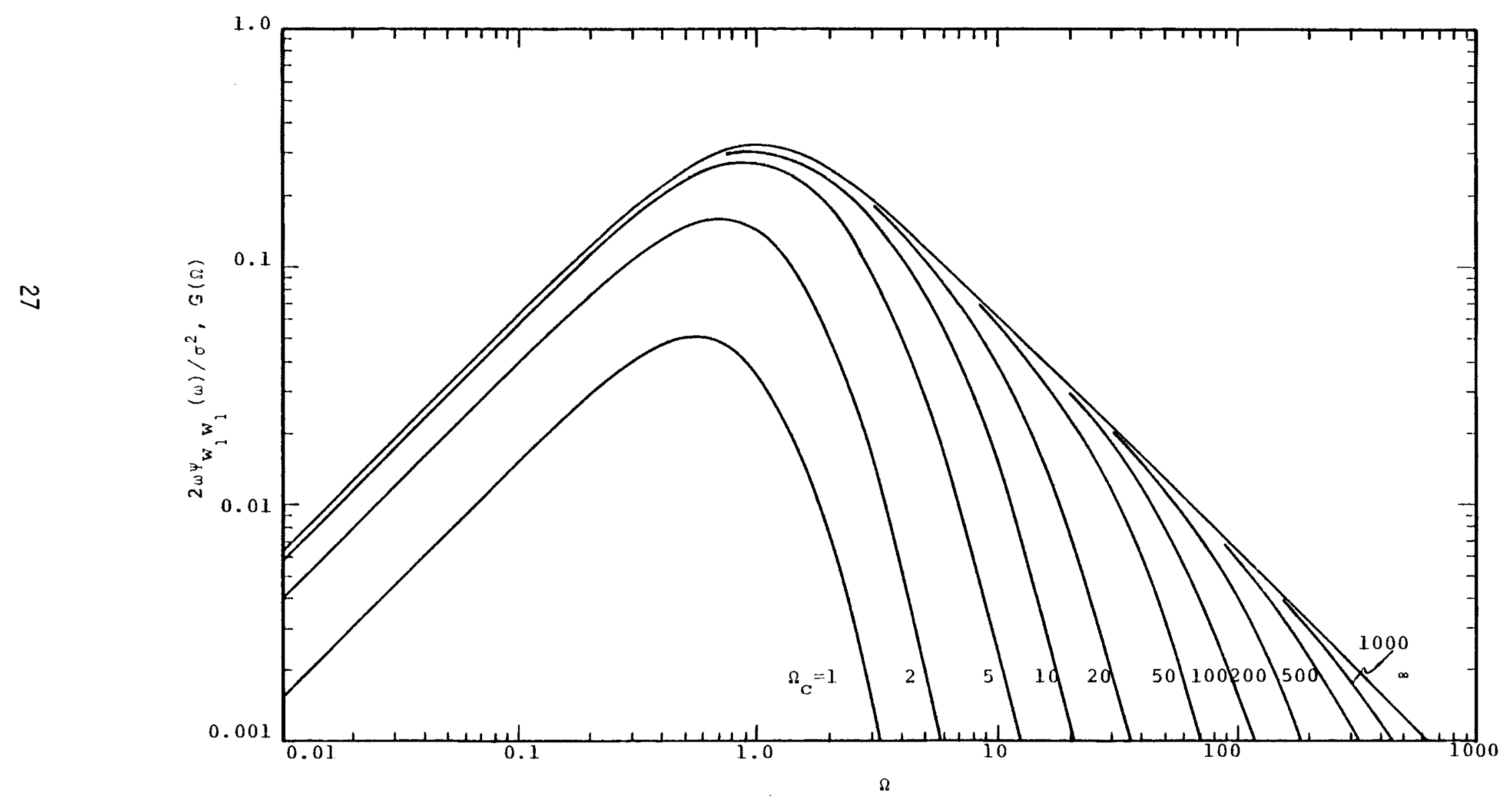

Figure 6. Longitudinal velocity component frequency spectra and function $G(\Omega)$. 


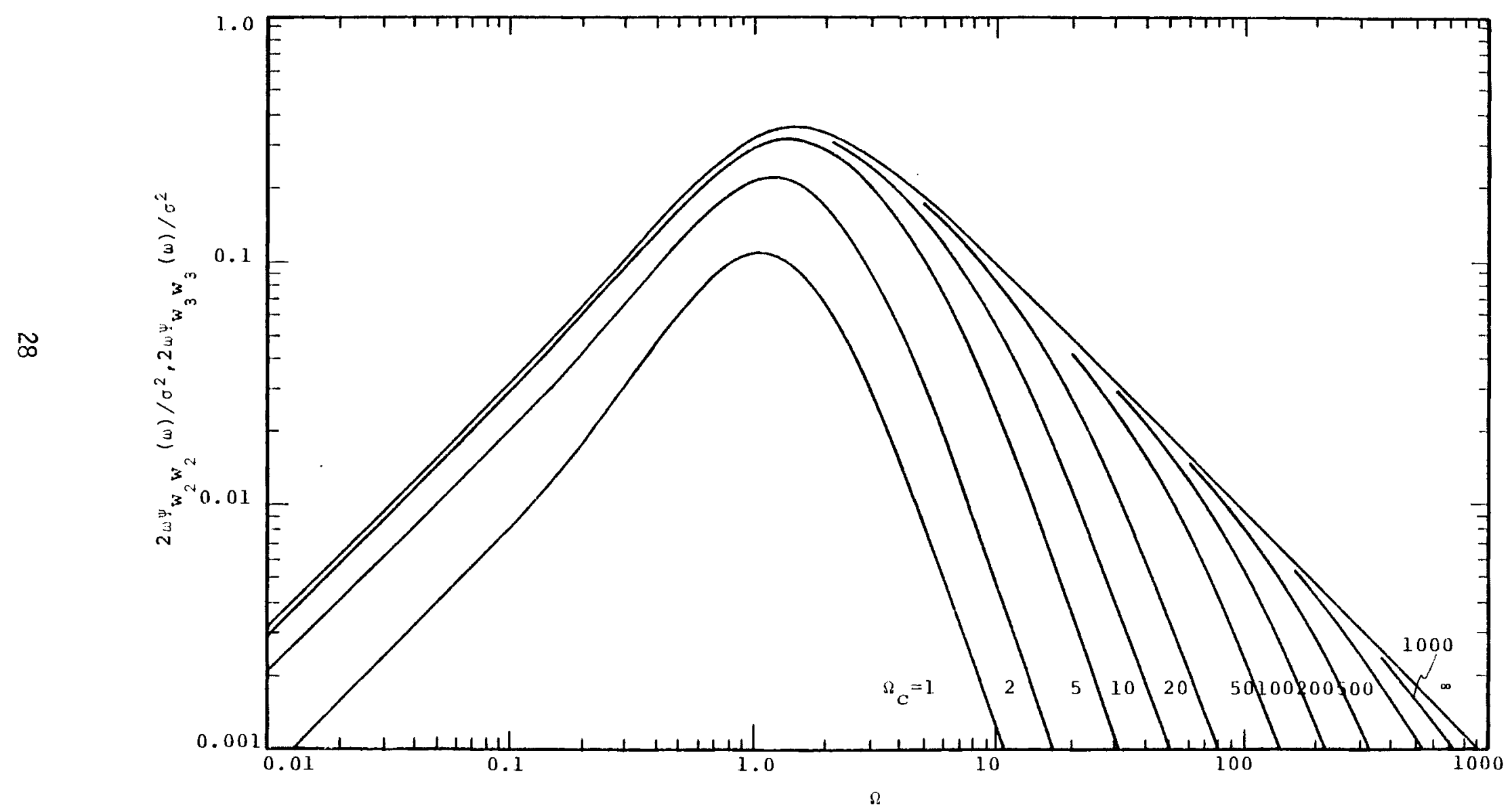

Figure 7. Lateral and vertical velocity component frequency spectra. 


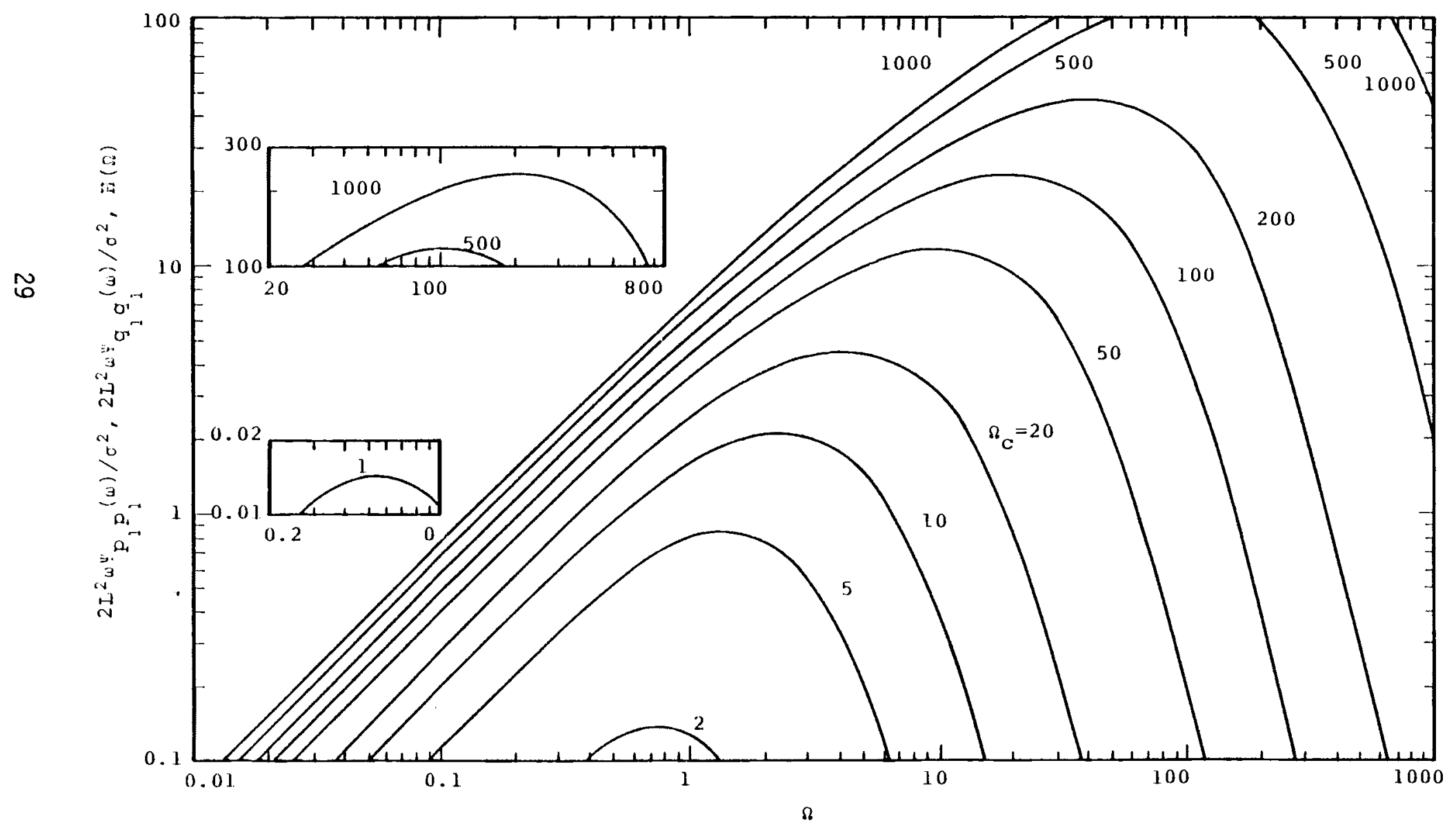

Figure 8. $p_{1}$ and $q_{1}$ frequency spectra and function $H(\Omega)$. 


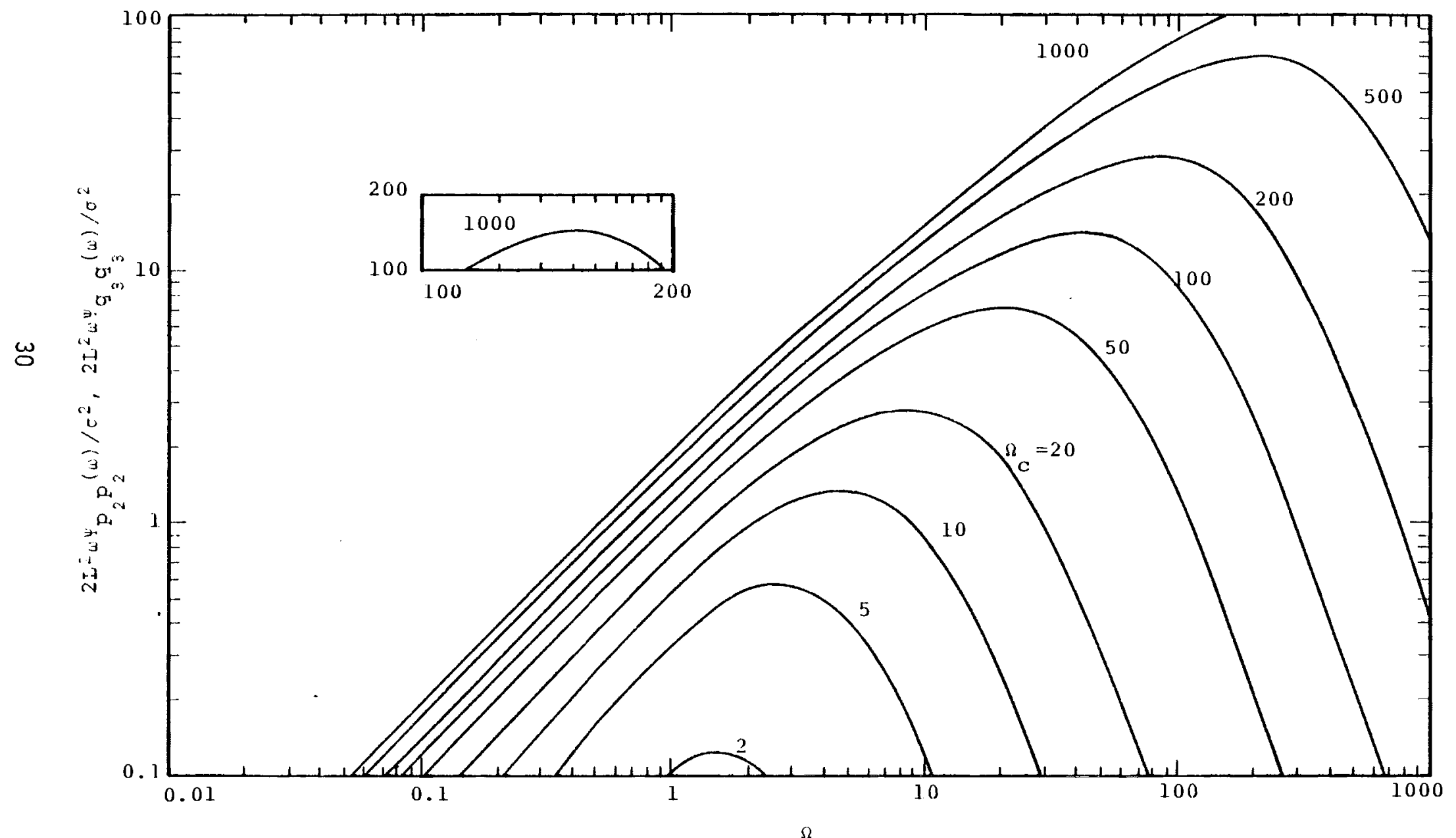

Finure 9. $\quad p_{2}$ and $a_{3}$ frequency spectra 


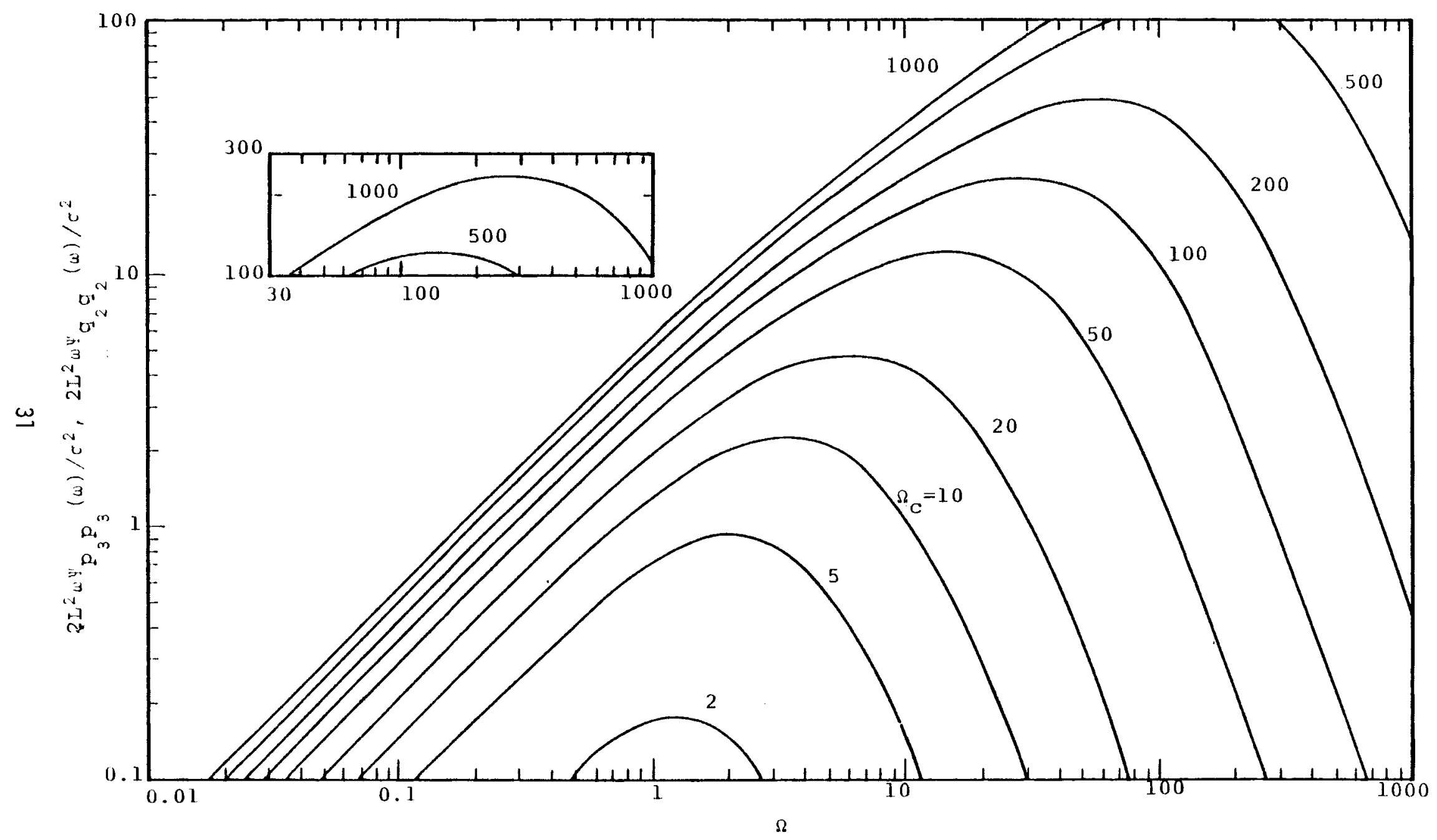

Fiqure 10. $p_{3}$ and $a_{2}$ frequency spectra 


\section{LINEAR SYSTEMS}

In this section we indicate the manner in which two-point, two-time covariance statistics might be used in WECS design. The discussion is not intended to be an exhaustive treatment of linear structural dynamics, but rather is intended to show how the covariance statistics might enter a response anlysis. A typical approach in system response analysis is to partition a structure, airfoil, etc., into nodes or strips whereby aerodynamic forces are applied and equations of motion are written for each node or strip. Furthermore, the equations are oftentimes linearized and dynamic responses are calculated with spectral density techniques. The linearized equations of motion might then look like

$$
L_{i k} \xi_{k}(t)=A_{i m}^{(s)} v_{s}\left(t ; r_{m}, a_{m}\right)
$$

where $\xi_{k}(t)$ is the $k^{\text {th }}$ dynamic variable of the system, and $L_{i k}$ is a linear operator of the form

$$
L_{i k}=E_{i k}^{(\nu)} \frac{d^{\nu}}{d t^{\nu}}+E_{i k}^{(\nu-1)} \frac{d^{\nu}-1}{d t^{\nu}-1}+\ldots+E_{i k}^{(0)} .
$$

The matrices $E_{i k}^{(v)}, v=1,2, \ldots$ and $A_{i m}^{(\nu)}$ with $s=1,2,3$ depend on the structural mass, stiffness, damping and aerodynamic characteristics. The operator $L_{i k}$ is a square $N \times N$ matrix operator with $N$ equal to the number of dynamic variables of the system, i.e., $q_{k}, k=1,2, \ldots N$. For each value of $s$ the matrix $A_{i m}^{(s)}$ is a $N \times M$ matrix with $M$ equal to the number of nodes or strips.

\section{FOURIER TRANSFORMS}

Let us assume that a Fourier transform exists for Equation (70), so that

$$
\hat{L}_{i k}(\omega) d z_{\xi_{k}}(\omega)=A_{i m}^{(p)} d z_{v_{p}}\left(\omega ; r_{m}, \alpha_{m}\right)
$$


where the $\xi_{k}$ and $v_{k}$ are related to the Fourier amplitudes ( $d z$ ) as follows:

$$
x(t)=\int_{-\infty}^{\infty} e^{i \omega t} d z(\omega)
$$

and

$$
\hat{L}_{i k}(\omega)=E_{i k}^{(\nu)}(i \omega)^{\nu}+E_{i k}^{(\nu-1)}(i \omega)^{\nu}-1+\ldots+E_{i k}^{(0)} .
$$

Multiplication of Equation (72) evaluated at frequency $\omega_{1}$ by the complex conjugate of Equation (72) evaluated at $\omega_{2}$ yields

$$
\begin{aligned}
& \hat{L}_{i k}\left(\omega_{1}\right) \hat{L}_{j n}^{\star}\left(\omega_{2}\right) \Psi_{\xi_{k} \xi_{n}}\left(\omega_{1}, \omega_{2}\right)= \\
& A_{i k}^{(s)} A_{j n}^{(m)_{\Psi_{v}} v_{s} v_{m}}\left(\omega_{1}, w_{2} ; r_{k}, r_{n}, \alpha_{k}, \alpha_{n}\right)
\end{aligned}
$$

where

$$
\Psi_{A B}\left(\omega_{1}, \omega_{2}\right) d \omega_{1} d \omega_{2}=\left\langle d Z_{A}\left(\omega_{1}\right) d z_{B}^{*}\left(\omega_{2}\right)\right\rangle .
$$

The quantity $\Psi_{A B}\left(\omega_{1}, \omega_{2}\right)$ is the double frequency (generalized) power spectrai density function.

It can be shown that

$$
R_{A B}\left(t_{1}, t_{2}\right)=\int_{-\infty}^{\infty} \Psi_{A B}\left(\omega_{1}, \omega_{2}\right) e^{-i\left(\omega_{1} t_{1}-\omega_{2} t_{2}\right)} d \omega_{1} d \omega_{2}
$$

where

$$
R_{A B}\left(t_{1}, t_{2}\right)=\left\langle A\left(t_{1}\right) B\left(t_{2}\right)\right\rangle .
$$


Furthermore,

$$
{ }_{A B}\left(\omega_{1}, \omega_{2}\right)=\frac{1}{(2 \pi)^{2}} \int_{-\infty}^{\infty} R_{A B}\left(t_{1}, t_{2}\right) e^{i\left(\omega_{1} t_{1}-\omega_{2} t_{2}\right)} d t_{1} d t_{2} .
$$

These equations are analogous to the Wiener-Khintchine Theorem in the stationary case. Note that $\Psi_{A B}\left(\omega_{1}, \omega_{2}\right)$ exists if $R_{A B}\left(t_{1}, t_{2}\right)$ is absolutely integrable. For further details see Bendat and Piersol (1971) or Lin (1967).

We now set $t_{1}=t$ and $t_{2}=t+\tau$, so that Equation (77) can be written as

$$
R_{A B}(\tau ; t)=\int_{-\infty}^{\infty}{ }_{A B}\left(\omega_{1}, \omega_{2}\right) e^{-j\left(\omega_{1}-\omega_{2}\right) t} e^{i \omega_{2} \tau} d \omega_{1} d \omega_{2}
$$

where the covariance function, is now a function of $\tau$ and $t$, i.e., we construct the covariance of $A$ at time $t$ and $B$ at time $t+\tau$. For stationary processes the covariance does not depend on time $t$. The only way this can be the case is for the double frequency power spectral density function to be of the form

$$
\Psi_{A B}\left(\omega_{1}, \omega_{2}\right)=\Psi_{A B}\left(\omega_{2}\right) \delta\left(\omega_{2}-\omega_{1}\right)
$$

i.e., zero spectral density for $\omega_{1} \neq \omega_{2}$. However, if we are dealing with nonstationary turbulence inputs and associated nonstationary responses, $\Psi_{A B}\left(\omega_{1}, \omega_{2}\right)$ can take on nonzero values for $\omega_{1} \neq \omega_{2}$.

Equation (75) can be solved to obtain the generalized power spectral density functions $\Psi_{\xi_{k} \xi_{n}}\left(\omega_{1}, \omega_{2}\right)$ of the response variables in terms of generalized two-point power spectral density functions of the turbulence. Equation (77) can then be used to obtain response covariances.

Another approach to describing spectral structure of nonstationary processes is to Fourier transform Equation (80) with respect to $\tau$, so that

$$
{ }_{A B}(\omega, t)=\frac{1}{2 \pi} \int_{-\infty}^{\infty} R_{A B}(\tau ; t) e^{-i \omega \tau} d \tau .
$$


Substitution of Equation (80) into Equation (82) yields

$$
{ }_{A B}(\omega ; t)=\quad \int_{-\infty}^{\infty} A B\left(\omega_{1}, \omega_{2}\right) \delta\left(\omega_{2}-\omega\right) e^{-i\left(\omega_{1}-\omega_{2}\right) t} t_{d \omega_{1}} d \omega_{2}
$$

or

$$
\Phi_{A B}(\omega ; t)=\int_{-\infty}^{\infty}{ }_{A B}\left(\omega_{1}, \omega\right) e^{-i\left(\omega_{1}-\omega\right) t_{d \omega_{1}} .}
$$

This defines the frequency-time spectral density function. For stationary processes

$$
\Psi_{A B}\left(\omega_{1}, \omega\right)=\Psi_{A B}\left(\omega_{1}\right) \delta\left(\omega-\omega_{1}\right)
$$

so that Equation (84) reduces to

$$
\Phi_{A B}(\omega, t)=\Psi_{A B}(\omega)
$$

Thus, the frequency-time spectral density function concept is a generalization of the traditional spectral decomposition theory for stationary processes.

GENERALIZED TURBULENCE SPECTRAL DENSITIES

If the theory enunciated in the previous section is applicable to WECS design, i.e., the WECS can be represented as a linear system, then we require the Fourier transform of Equation (15), so that

$$
\begin{gathered}
{ }_{v_{i} v_{j}}\left(\omega_{1}, \omega_{2} ; r_{1}, r_{2}, \alpha_{1}, \alpha_{2}\right)=\Psi_{S_{i j}}\left(\omega_{1}, \omega_{2} ; r_{1}, r_{2}, \alpha_{1}, \alpha_{2}\right) \\
+\psi_{N_{i j}}\left(\omega_{1}, \omega_{2} ; r_{1}, r_{2}, \alpha_{1}, \alpha_{2}\right) .
\end{gathered}
$$

The quantities $r_{1}, r_{2}, \alpha_{1}$ and $\alpha_{2}$ can be replaced with $r_{k}, r_{n}, \alpha_{k}$ and $\alpha_{n}$ 
(see Equation (75)) and the subscripts can take on integer values as discussed in the previous section. Fourier transformation of Equations (35 and 36) yields generalized spectral density functions for the stationary and nonstationary parts of the two-point, two-time turbulence covariance matrix, so that

$$
\begin{aligned}
& { }^{\Psi} S_{i j}\left(\omega_{1}, \omega_{2} ; r_{1}, r_{2}, \alpha_{1}, \alpha_{2}\right)=\left\{{ }^{w_{i}} w_{j}\left(\omega_{2}\right)-\frac{1}{2}\left(r_{1}^{2}+r_{2}^{2}\right) \cdot\right. \\
& {\left[\psi_{p_{i}} p_{j}\left(\omega_{2}\right)+\psi_{q_{j}} q_{j}\left(\omega_{2}\right)\right]+\frac{r_{1} r_{2}}{2}\left[\left(\psi_{p_{i}} p_{j}\left(\omega_{2}-\omega_{0}\right)+\right.\right.} \\
& \left.\Psi_{i} q_{j}\left(\omega_{2}-\omega_{0}\right)\right) e^{i\left(\alpha_{1}-\alpha_{2}\right)}+\left(\Psi_{p_{i} p_{j}}\left(\omega_{2}+\omega_{0}\right)+\right. \\
& \left.\left.\left.\Psi_{i} q_{j}\left(\omega_{2}+\omega_{0}\right)\right) e^{-i\left(\alpha_{1}-\alpha_{2}\right)}\right]\right\} \delta\left(\omega_{2}-\omega_{1}\right) \\
& { }^{\Psi} N_{i j}\left(\omega_{1}, \omega_{2} ; r_{1}, r_{2}, \alpha_{1}, \alpha_{2}\right)=\frac{1}{2}\left\{r_{2} \Psi_{w_{i}} p_{j}\left(\omega_{2}\right) e^{i \alpha_{2}}\right. \\
& \left.-r_{1} w_{i} p_{j}\left(\omega_{2}-\omega_{0}\right) e^{i \alpha_{1}}\right\} \delta\left(\omega_{2}-\omega_{1}-\omega_{0}\right)+\frac{1}{2}\left\{r_{2} w_{i} p_{j}\left(\omega_{2}\right) e^{-i \alpha_{2}}\right. \\
& \left.-r_{1} w_{w_{i}} p_{j}\left(\omega_{2}+\omega_{0}\right) e^{-i \alpha_{1}}\right\} \delta\left(\omega_{2}-\omega_{1}+\omega_{0}\right)-\frac{i}{2}\left\{r_{2} w_{i} q_{j}\left(\omega_{2}\right) e^{i \alpha_{2}}\right. \\
& \left.-r_{1} w_{i} q_{j}\left(\omega_{2}-\omega_{0}\right) e^{i \alpha_{1}}\right\} \delta\left(\omega_{2}-w_{1}-\omega_{0}\right)+\frac{i}{2}\left\{r_{2} w_{i} q_{j}\left(\omega_{2}\right) e^{-i \alpha_{2}}\right. \\
& \left.-r_{1} w_{i} q_{j}\left(\omega_{2}+\omega_{0}\right) e^{-i \alpha_{1}}\right\} \delta\left(\omega_{2}-\omega_{1}+\omega_{0}\right)+\frac{i}{4}\left\{r_{1}^{2} q_{i} p_{j}\left(\omega_{2}-2 \omega_{0}\right) e^{i 2 \alpha_{1}}\right.
\end{aligned}
$$




$$
\begin{aligned}
& \left.+r_{2}^{2} q_{i} p_{j}\left(\omega_{2}\right) e^{i 2 \alpha_{2}}-2 r_{1} r_{2} q_{i} p_{j}\left(\omega_{2}-\omega_{0}\right) e^{i\left(\alpha_{1}+\alpha_{2}\right)}\right\} \delta\left(\omega_{2}-\omega_{1}-2 \omega_{0}\right)
\end{aligned}
$$

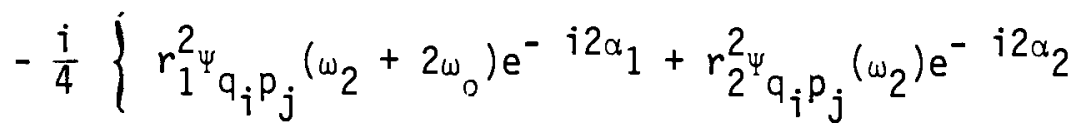

$$
\begin{aligned}
& \left.-2 r_{1} r_{2} \Psi q_{i} p_{j}\left(\omega_{2}+\omega_{0}\right) e^{-i\left(\alpha_{1}+\alpha_{2}\right)}\right\} \delta\left(\omega_{2}-\omega_{1}+2 \omega_{0}\right) \\
& +\frac{1}{8}\left\{r_{1}^{2}\left(\Psi_{i} q_{j}\left(\omega_{2}-2 \omega_{0}\right)-\Psi_{p_{j} p_{j}}\left(\omega_{2}-2 \omega_{0}\right)\right) e^{i 2 \alpha_{1}}\right.
\end{aligned}
$$

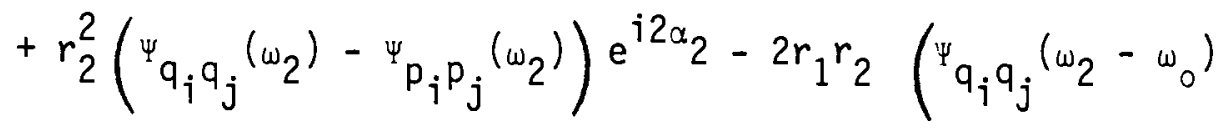

$$
\begin{aligned}
& \left.\left.-\Psi_{p_{i} p_{j}}\left(\omega_{2}-\omega_{0}\right)\right) e^{i\left(\alpha_{1}+\alpha_{2}\right)}\right\} \delta\left(\omega_{2}-\omega_{1}-2 \omega_{0}\right) \\
& +\frac{1}{8}\left\{r_{1}^{2}\left(\Psi_{i} q_{j}\left(\omega_{2}+2 \omega_{0}\right)-\Psi_{p_{j} p_{j}}\left(\omega_{2}+2 \omega_{0}\right)\right) e^{-i 2 \alpha_{1}}\right. \\
& +r_{2}^{2}\left(\Psi_{j} q_{j}\left(w_{2}\right)-\Psi_{p_{j} p_{j}}\left(w_{2}\right)\right) e^{-i 2 \alpha_{2}} \\
& \left.-2 r_{1} r_{2}\left(\Psi_{j} q_{j}\left(\omega_{2}+\omega_{0}\right)-\Psi_{p_{j} p_{j}}\left(\omega_{2}+\omega_{0}\right)\right) e^{-i\left(\alpha_{1}+\alpha_{2}\right)}\right\} \delta\left(\omega_{2}-\omega_{1}+2 \omega_{0}\right) .
\end{aligned}
$$

Equations(88 and 89) permit the calculation of the two-point generalized power spectral density of turbulence for WECS linear response analyses upon specification of $E(k), r_{1}, r_{2}, \alpha_{1}, \alpha_{2}$ and $\omega_{0}$. In the case of the Dryden spectral model the auto- and cross-spectral density functions given by Equations (58 through 69) can be evaluated at the frequencies $\omega_{2}, \omega_{2} \pm \omega_{0}$ and $\omega_{2} \pm 2 \omega_{0}$ and substituted into Equations ( 88 and 89 ) and the spectrum given by Equation ( 87 ) is immediately specified. 
The various terms in Equations ( 88 and 89 ) are nonzero on the lines

$$
\omega_{2}-\omega_{1}-n \omega_{0}=0, n=0,1,2
$$

in the $\left(\omega_{1}, \omega_{2}\right)$-plane (see Figure 11) and vanish everywhere else. As we add more terms to the Taylor series expansion of the turbulence velocity field the number of lines on which the generalized turbulence spectral densities are nonzero increase, i.e., $n=3,4, \ldots$. At $\omega_{0}=0$ all of the lines collapse onto the line $\omega_{2}-\omega_{1}=0$ and the generalized turbulence spectral densities are of the form given Equation (81) appropriate for a stationary process. As $\omega_{0}$ increases away from zero the lines in Figure 11 separate. The spacing between these lines can be thought of as being a measure of the degree to which the turbulence velocity statistics depart from statistical stationary conditions.

If $i=j$ the coefficient of the delta function in Equation (88) is nonzero. If $\alpha_{1}=\alpha_{2}$ this coefficient is real and if $\alpha_{1} \neq \alpha_{2}$ the coefficient is complex implying a phase shift between the turbulence components at points $\left(r_{1}, \alpha_{1}\right)$ and $\left(r_{2}, a_{2}\right)$. It is interesting to note that this phase shift vanishes when $\alpha_{1}=\alpha_{2}$ for all values of $r_{1}$ and $r_{2}$. If $i \neq j$ the cross-spectral density functions on the right side of Equation (88) vanish identically so that $\Psi_{S}$ vanishes.

We scale the position coordinates $r_{1}$ and $r_{2}$ with WECS blade length $c$. The lowest order term in Equation (88) is $c^{0}$ with the next highest order being $c^{2}$ It can be shown that terms above second-order in $\Psi_{S}$ form a series involving even ascending powers of $c$, i.e., $c^{q}, q=4,6, \ldots$

We now turn our attention to the nonstationary part of the generalized turbulence spectral densities. For ease of discussion we number each delta function term in Equation (89) from left to right from 1 through 8 . If $i=j$ terms 1 through 6 vanish. In this case the lead term is of order $c^{2}$. Furthermore, it can be shown that the remaining terms form a series involving ascending even powers of $c$, i.e., $c^{q}, q=4,6, \ldots$ If $i \neq j$ terms 7 and 8 vanish and terms 1 and 2 are nonzero for $i=1$ and $j=2$, terms 3 and 4 are nonzero for $i=1$ and $j=3$, and terms 5 and 6 are nonzero for $i=2$ and $j=3$. Thus, for $i=1$ and $j=2$ and $i=1$ and $j=3$ the lead term in Equation (89) is of order $c^{1}$ and for $i=2$ and $j=3$ the lead term is of order $c^{2}$. It can be shown for the case of the former the higher order terms form a series involving 


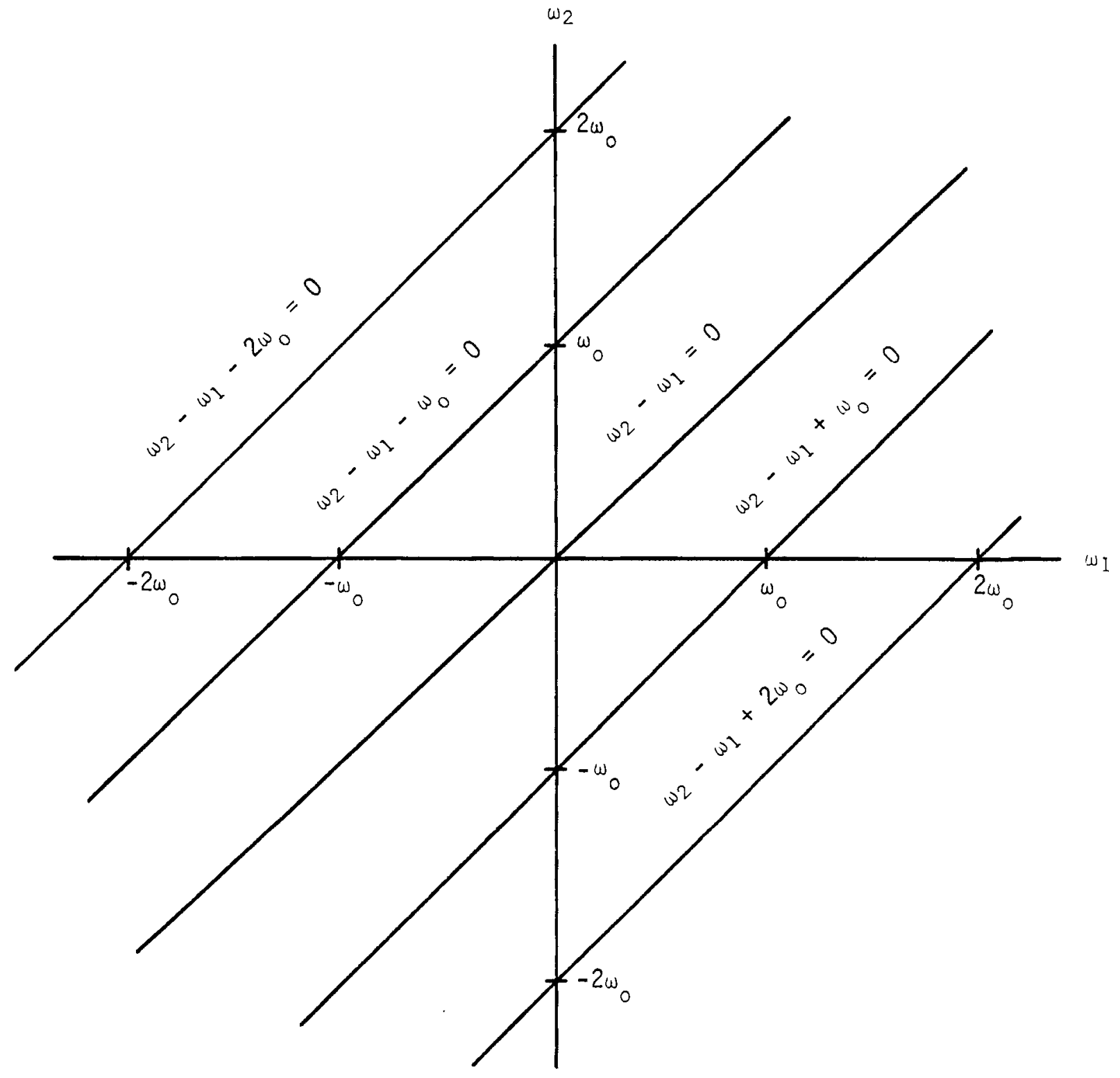

Figure 11. The $\left(\omega_{1}, \omega_{2}\right)$-plane. Each line corresponds to a contribution to the generalized turbulence spectral density function. The spacing between iines is a measure of the degree to which the wind process following the WECS biade eiements is a nonstationary process. 
ascending odd powers of $c$, i.e., $c^{q}, q=3,5, \ldots$ and in the latter the remaining terms form a series involving ascending even powers of $c$, i.e., $c^{q}, q=4$, $6, \ldots$

FREQUENCY-TIME SPECTRAL DENSITIES

Application of Equation (84) to Equation (87) yields the frequency-time spectral density function of the turbulence velocity components following the WECS blade elements, namely

$$
\begin{gathered}
\Phi_{v_{i} v_{j}}\left(\omega ; t, r_{1}, r_{2}, \alpha_{1}, \alpha_{2}\right)=\Phi_{S_{i j}}\left(\omega ; t, r_{1}, r_{2}, \alpha_{1}, \alpha_{2}\right) \\
+\Phi_{N_{i j}}\left(\omega, t, r_{1}, r_{2}, \alpha_{1}, \alpha_{2}\right)
\end{gathered}
$$

where

$$
\begin{gathered}
{ }_{\Phi_{i j}}\left(\omega ; r_{1}, r_{2}, \alpha_{1}, \alpha_{2}\right)=\Psi_{w_{i} w_{j}}(\omega)-\frac{1}{2}\left(r_{1}^{2}+r_{2}^{2}\right) \cdot \\
{\left[\Psi_{p_{i} p_{j}}(\omega)+{ }^{q_{i}} q_{j}(\omega)\right]+\frac{r_{1} r_{2}}{2}\left[\left(\Psi_{p_{i} p_{j}}\left(\omega-\omega_{0}\right)+\right.\right.} \\
\left.{ }_{q_{i} q_{j}}\left(\omega-\omega_{0}\right)\right) e^{i\left(\alpha_{1}-\alpha_{2}\right)+\left(\Psi_{p_{j} p_{j}}\left(\omega-\omega_{0}\right)+\right.} \\
\left.\left.{ }_{q_{i} q_{j}}\left(\omega+\omega_{0}\right)\right) e^{-i\left(\alpha_{1}-\alpha_{2}\right)}\right]
\end{gathered}
$$

and 


$$
\begin{aligned}
& \Phi_{N_{i j}}\left(\omega ; t, r_{1}, r_{2}, \alpha_{1}, \alpha_{2}\right)=r_{2} \Psi_{w_{i}} p_{j}(\omega) \cos \left(\omega_{0} t+\alpha_{2}\right)+r_{2} \Psi_{w_{j} q_{j}}(\omega) \sin \left(\omega_{0} t+\alpha_{2}\right) \\
& -\frac{1}{2}\left\{r_{1} w_{j} p_{j}\left(\omega-\omega_{0}\right) e^{i \alpha 1} e^{i \omega_{0} t}+r_{1} \Psi_{w_{j}} p_{j}\left(\omega+\omega_{0}\right) e^{-i \alpha_{1} e^{-i \omega_{0} t}}\right\} \\
& +\frac{j}{2}\left\{r_{1} \Psi_{w_{j} q_{j}}\left(\omega-\omega_{0}\right) e^{i \alpha 1} e^{i \omega_{0} t}+r_{1} \Psi_{w_{j} q_{j}}\left(\omega+\omega_{0}\right) e^{-i \alpha} 1^{-i \omega_{0} t}\right\} \\
& -2 r_{2}^{2} q_{j} p_{j}(\omega) \sin \left(2 \omega_{o} t+\alpha_{2}\right) \\
& +\frac{i}{4}\left\{r_{1}^{2} q_{j} p_{j}\left(\omega-\omega_{0}\right) e^{i 2 \alpha} 1_{e}^{i 2 \omega_{0} t}-r_{1}^{2} q_{j} p_{j}\left(\omega+2 \omega_{0}\right) e^{-i 2 \alpha 1} e^{-i 2 \omega_{0} t}\right\}
\end{aligned}
$$

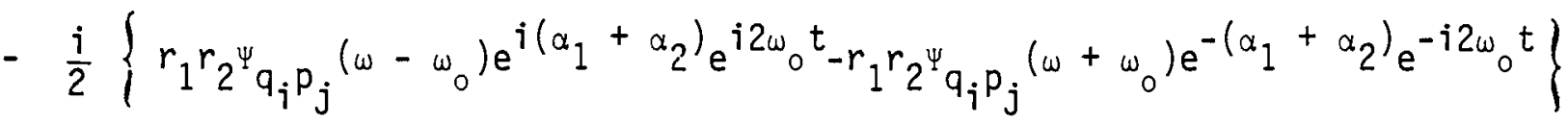

$$
\begin{aligned}
& +\frac{r_{2}^{2}}{4}\left(\psi_{q_{j} q_{j}}(\omega)-\psi_{p_{j} p_{j}}(\omega)\right) \cos \left(2 \omega_{0} t+2 \alpha_{2}\right) \\
& +1 / 8\left\{r_{1}^{2}\left(\Psi_{i} q_{j}\left(\omega-2 \omega_{0}\right)-\Psi_{p_{j} p_{j}}\left(\omega-2 \omega_{0}\right)\right) e^{i 2 \alpha 1_{e} e^{i 2 \omega_{0} t}}\right. \\
& +r_{1}^{2}\left(\psi_{q_{j} q_{j}}\left(\omega+2 \omega_{0}\right)-\Psi_{p_{j} p_{j}}\left(\omega+2 \omega_{0}\right)\right) e^{-i 2 \alpha 1 e^{-2 \omega_{0} t}} \\
& -\frac{1}{4}\left\{r_{1} r_{2}\left(\psi_{q_{j}} q_{j}\left(\omega-\omega_{0}\right)-\Psi_{p_{i} p_{j}}\left(\omega-\omega_{0}\right)\right) e^{i\left(\alpha_{1}+\alpha_{2}\right) e^{i 2 \omega_{0} t}}\right. \\
& +r_{1} r_{2}\left(\Psi_{j} q_{j}\left(\omega+\omega_{0}\right)-\Psi_{p_{j} p_{j}}\left(\omega+\omega_{0}\right)\right) e^{-i\left(\alpha_{1}+\alpha_{2}\right) e^{-i 2 \omega_{0} t}}
\end{aligned}
$$

(93)

42 
The function $\Phi_{S}$ does not depend on time because $S_{v_{i j}} v_{j}$ is the stationary part of the two-point turbulence velocity component covariance function. The function $\Phi_{N_{i j}}$ clearly depends on time. This function contains sinusoidal variations in time with frequencies $t_{\omega_{0}}$ and $+2 \omega_{0}$. If terms beyond secondorder were included in the original Taylor series of the gust velocity component field then additional terms with sinusoidal variations in time with frequencies $\pm n \omega_{0}, n=3,4, \ldots$ would be contained in Equation (93). 



\section{AN EXAMPLE OF CALCULATED WIND SPECTRUM EXPERIENCED BY A ROTATT ING BLADE}

To provide an example we shall consider spectra following a single blade element, i.e., $r_{1}=r_{2}=r$, and $\alpha_{1}=\alpha_{2}=\alpha$, for $i=j=1$. This corresponds to the spectrum of turbulence for the component orthogonal to the disk of rotation. We select this case because measurements are available by which we can assess the fidelity of the theory presented herein. In this case the generalized turbulence spectral densities are given by

$$
\begin{aligned}
& { }_{S_{11}}\left(\omega_{1}, \omega_{2} ; r, r, \alpha, \alpha\right)=\left\{\Psi_{w_{1} w_{1}}\left(\omega_{2}\right)+r^{2}\left[\Psi_{p_{1} p_{1}}\left(\omega_{2}-\omega_{0}\right)\right.\right. \\
& \left.\left.+\Psi_{p_{1} p_{1}}\left(\omega_{2}+\omega_{0}\right)-2 \Psi_{p_{1} p_{1}}\left(\omega_{2}\right)\right]\right\} \delta\left(\omega_{2}-\omega_{1}\right) \\
& { }^{\Psi_{N}}\left(\omega_{11}, \omega_{2} ; r, r, \alpha, \alpha\right)=0 \quad .
\end{aligned}
$$

The corresponding frequency-time spectral density function is given by

$$
\begin{gathered}
\Phi_{11}(\omega, t ; r, r, \alpha, \alpha)=\Psi_{w_{1} w_{1}}(\omega)+r^{2}\left[\Psi_{p_{1} p_{1}}\left(\omega-\omega_{0}\right)\right. \\
\left.+\Psi_{p_{1} p_{1}}\left(\omega+\omega_{0}\right)-2 \Psi_{p_{1} p_{1}}(\omega)\right] .
\end{gathered}
$$

Equation (96) can be written in nondimensional form namely

$$
\begin{gathered}
2 \omega \Phi_{S_{11}}(\omega, t ; r, r, \alpha, \alpha)=\frac{2 \omega w_{1} w_{1}(\omega)}{\sigma^{2}} \\
+\left(\frac{r}{L}\right)^{2}\left\{\frac{\omega}{\omega-\omega_{0}} \frac{2 L^{2}\left(\omega-\omega_{0}\right)}{\sigma^{2}} \Phi_{p_{1} p_{1}}\left(\omega-\omega_{0}\right)+\frac{\omega}{\omega+\omega_{0}} \frac{2 L^{2}\left(\omega+\omega_{0}\right)}{\sigma^{2}} \Phi_{p_{1} p_{1}\left(\omega+\omega_{0}\right)}\right. \\
\left.-2 \frac{2 L^{2} \omega{ }^{2} p_{1} p_{1}(\omega)}{\sigma^{2}}\right\}
\end{gathered}
$$


The nondimensional spectra on the right hand side of Equation (97) are universal functions of $\Omega$ and $\Omega_{c}$ and were presented earlier for the Dryden spectral turbulence mode 1 . Measured spectra are usually represented in terms of the cyclic frequency

$$
n=\frac{\omega}{2 \pi}
$$

so that for turbulence models characterized by an integral scale L, like in the von Karman and Dryden models,

$$
\Omega=\frac{w L}{u_{0}}=2 \pi \frac{L}{z_{h}} \frac{n z_{h}}{u_{0}}
$$

where

$$
z_{h}=\text { WECS hub height. }
$$

Furthermore,

$$
\frac{n \Phi^{\prime}(n)}{\sigma^{2}}=\frac{\omega \Phi(\omega)}{\sigma^{2}}
$$

where $\Phi(n)$ and $\Phi(\omega)$ are spectra of the same randon function in the cyclic and radian frequency domains. Thus, upon specification of $L / z_{h}$ and $\Omega_{C}$, which is discussed below, we can calculate the nondimensional spectrum ${ }^{n \Phi_{S}} S_{11}(n, t ; r, r, \alpha, \alpha) / \sigma^{2}$ as a function of $n z_{h} / u_{0}$.

At the WECS hub $(r=0)$ the spectrum is given by the lead term in the Taylor series expansion of the velocity field. If the WECS does not rotate $\left(\omega_{0}=0\right)$ then the second- and higher-order terms also vanish (see Equation (97). This means the spectrum at the hub can be applied anywhere in the $(y, z)$ plane for the nonrotating case. In the rotating case away from the WECS hub the second-order terms do not necessarily vanish because the time history of turbulence as "seen" by a WECS blade element will be different from that experienced in the nonrotating case. This results from the fact that the trajectory of a blade element through the mean atmospheric flow state is a helix 
To obtain the spectrum of turbulence following a blade element we must select values of $\Omega_{c}$ and $\mathrm{r} / \mathrm{L}$. However, the use of a truncated Taylor series of the turbulent flow implies that we must place restrictions on the permissible values of $\Omega_{c}$ and $r / L$. In the case of the spectrum for $i=j=1$ all of the terms in the Taylor series expansion, Equation (3), which involve oddorder derivatives vanish. To neglect the fourth- and higher-order terms in Equation (3) and hence in the spectrum, Equation (96), we must require

$$
\frac{x_{2}^{2}}{2} \frac{\partial^{2} u_{1}}{\partial x_{2}^{2}}, \frac{x_{3}^{2}}{2} \frac{\partial^{2} u_{1}}{\partial x_{3}^{2}}, x_{2} x_{3} \frac{\partial^{2} u_{1}}{\partial x_{2} \partial x_{3}}>>\frac{x_{2}^{4}}{24} \frac{\partial^{4} u_{1}}{\partial x_{2}^{4}}, \frac{x_{3}^{4}}{24} \frac{\partial^{4} u_{1}}{\partial x_{3}^{4}}, \frac{x_{2}^{2} x_{3}^{2}}{4} \frac{\partial^{4} u_{1}}{\partial x_{2}^{2} \partial x_{3}^{2}}
$$

If we estimate the spatial derivatives as $\partial^{2} u_{1} / \partial x_{2}^{2} \sim \sigma / L^{2}$, etc, and then form ratios of corresponding fourth-order to second-order terms and demand the ratios to be very small compared to unity this yields a condition on the permissible values of $r / L$, i.e.

$$
\frac{x_{2}^{2} x_{3}^{2}}{4} \frac{\partial^{4} u_{1}}{\partial x_{2}^{2} \partial x_{3}^{2}} / x_{2} x_{3} \frac{\partial^{2} u_{1}}{\partial x_{2} \partial x_{3}} \simeq \frac{r^{4} \sigma}{4 L^{4}} / \frac{r^{2} \sigma}{L^{2}}=\frac{r^{2}}{4 L} \ll 1
$$

or

$$
\frac{r}{L} \ll 2 .
$$

If we accept values of $r / L$ which are no more than $10 \%$ of the right side of this inequality, then we must require

$$
\frac{r}{L} \lesssim 0.2
$$

We now derive a condition for $\Omega_{c}$. In view of truncation of the third- and higher-order terms in the Taylor series of the turbulence velocity field we must restrict the analysis to Fourier components with lateral and vertical wavelengths which are greater than or equal to twice the WECS diameter. This means we must require $\Omega_{c} \lesssim 2 \pi L / 4 c$, where $c$ is blade length. Let us choose $c / L \simeq 0.2$ consistent 
with Equation (103), so that we must require

$$
\Omega_{c} \leqslant 8
$$

We shall use select values of $\Omega_{c}$ to estimate the second-order contributions to the spectrum, i.e. the terms in the curly brackets in Equation (97). However, we shall use $\Omega_{C}=\infty$ to calculate the lead term in Equation (97), i.e. the spectrum density of the longitudinal component of turbulence of the hub. This will be conservative from an engineering standpoint.

Figure 12 is a sample calculation of the spectrum, Equation (97), for the Dryden turbulence model for the following parameters:

$$
\begin{gathered}
z_{h}=36 \mathrm{~m} \\
w_{0}=3.927 \mathrm{rad} \mathrm{sec}^{-1} \\
u_{0}=10 \mathrm{~m} \mathrm{sec}^{-1} \\
L=100 \mathrm{~m} \\
L / z_{h}=2.778 \\
r / L=0.25 \\
\Omega_{c}=0,5,7,8,10
\end{gathered}
$$

Our selected value of $r / L=0.25$ violates our criteria, Equation (103); however, this value of $r / L$ is sufficiently closed to 0.2 such that it is our opinion that the theory herein is still applicable. Our motivation for selecting $r / L=0.25$ is that experimental data is available by which we can test the theory herein. The solid line corresponds to the lead term in the Taylor series expansion, or the spectrum at the WECS hub. It was obtained by setting $\Omega_{c}=0$ in the second-order terms in the turbulence spectrum. The remaining spectra correspond to the spectrum at the WECS blade element located at $r / L=0.25$ for the various indicated values of $\Omega_{c}$. We have included the case $\Omega_{c}=10$. In this case the spectrum goes negative for a band of frequencies in the neighborhood of $n z_{h} / u_{0} \sim 0.2$. The negative values of spectrum can only be corrected by including fourth- and higher-order terms in the Taylor series expansion of the velocity field. We have included the case $\Omega_{c}=10$ to show that our criteria for $\Omega_{C}$ appears to work relative to delineating these regions of $\left(k_{2}, k_{3}\right)$ - space where fourth- and higher-order terms are needed in the analysis. The general features of the spectrum for $\Omega_{c} \neq 0$ are as follows: 


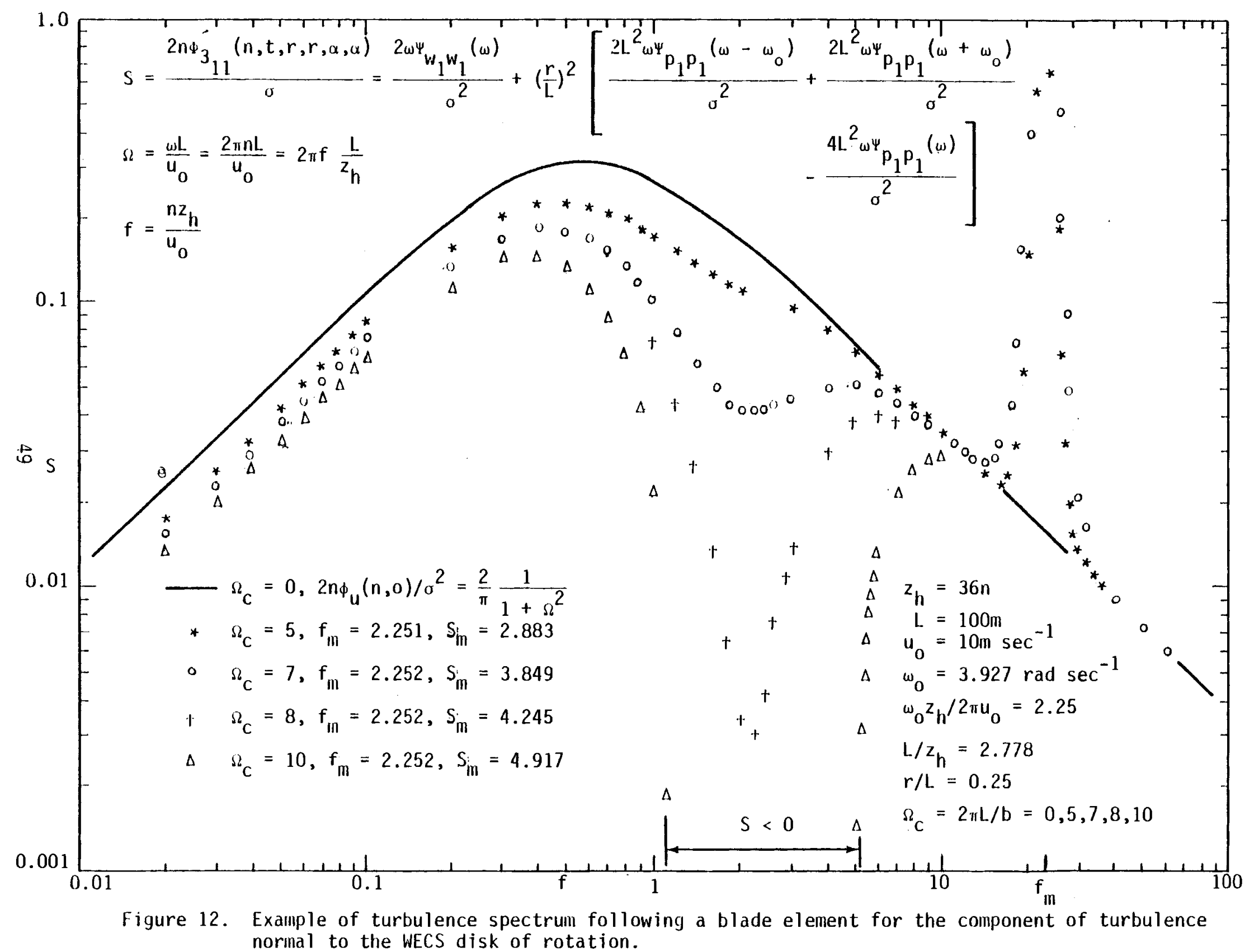


1) a relatively small energy deficit relative to the hub spectrum for $\left.0<n z_{h} / u_{0} \leqslant 0.4 ; 2\right)$ a substantial energy deficit in the region $0.4 \leqslant n z_{h} / u_{0} \lesssim 0.8 ; 3$ ) a pronounced spectral peak at frequency $n z_{h} / u_{0} \simeq 2.25$ corresponding to nondimensional cyclic WECS rotation frequency and 4) a return to the hub spectrum for frequencies larger than the hub frequency.

Connell (1980) has calculated spectra with experimental data acquired at the Pacific Northwest Laboratories, Richland, Washington, for conditions given by Equation (105). Except for the high frequency region beyond the spectral peak associated with the WECS rotation rate the theoretical spectra in this paper and those of Connell are essentially in agreement. The results are in best quantitative agreement with those of Connell for $\Omega_{c} \simeq 7$. The major discrepency between these results and those of Connell occur at frequencies beyond $n z_{h} / u_{0} \simeq 2.25$. Connell's observed spectrum increases like $n^{2}$ beyond the WECS rotation frequency. We attribute this discrepency to the failure to retain fourth- and higher-order terms in our analysis. Nevertheless, the truncated Taylor series expansion herein captures most, if not all, of the features observed by Connell for frequencies at and below the WECS rotation frequency. Inclusion of the fourth- and higher-order terms will produce spectral peaks essentially at integral WECS rotation frequencies, i.e., $2 \omega_{0}, 3 \omega_{0}$.

The theory presented herein does not include the contribution due to mean flow wind shear. This contribution to the $i=j=1$ spectrum is discussed in Appendix $C$. 


\section{CONCLUSIONS AND RECOMMENDATIONS}

Specification of covariance wind statistics following WECS blade elements is not straightforward. However, through the use of the simplifying assumptions of isotropic and homogeneous turbulence it has been possible to develop a second-order model of the covariance matrix, such that upon specification of the energy density spectrum function $E(\kappa)$ the nonstationary covariance statistics and associated spectra, for the turbulence velocity vector components following the WECS blade elements, are completely specified, wut witi accuracy wilich varies witn irequency of the spectral component. The central idea is that the turbulence velocity vector field can be expanded about the WECS hub with the result that the series expansion of the covariance matrix involves coefficients proportional to the covariance statistics of the gust velocity components and associated spatial derivatives to the same order as the highest term retained in the expansion. This expansion given by Equation (15) is general and can be applied to the anisotropic and vertically nonhomogeneous boundary layer as a logical next step. In this case we do not have a single function available, like $E(k)$ in the isotropic and homogeneous case, from which to derive the coefficients in the covariance expansion following the WECS blade elements. To develope a nonstationary gust covariance model for the anisotropic and vertically nonhomogeneous case we require the Eulerian field covariance matrix, namely

$$
R_{i j}\left(\tau, \xi_{2}, \xi_{3} ; x_{3}\right)=\left\langle v_{i}^{\prime}\left(t+\tau, x_{1}, x_{2}+\xi_{2}, x_{3}+\xi_{3}\right) v_{j}^{\prime}\left(t, x_{1}, x_{2}, x_{3}\right)\right\rangle
$$

to determine the coefficients in the gust covariance matrix following the WECS blade elements. Data is available for $R_{i j}\left(\tau, 0, \xi_{3}, x_{3}\right)$ and to a lesser degree for $R_{i j}\left(\tau, \xi_{2}, 0, x_{3}\right)$ for various micrometeorological tower sites. The Battelle Pacific Northwest Laboratories meteorological tower facility for WECS design criteria development provides the unique opportunity to acquire data for $R_{i j}\left(\tau, \xi_{2}, \xi_{3}, x_{3}\right)$ for simultaneous nonzero values of $\tau, \xi_{2}$, and $\xi_{3}$. In lieu of these gust statistics, working hypotheses might be used to construct the function $R_{i j}\left(\tau, \xi_{2}, \xi_{3}, x_{3}\right)$ from presently available Eulerian gust statistics to develope a gust covariance model following the WECS blade elements for the anisotropic and vertically nonhomogeneous boundary layer. 


\section{$\underline{\text { REFERENCES }}$}

Batchelor, G. K., 1969: The Theory of Homogeneous Turbulence. Cambridge University Press, Cambridge, $197 \mathrm{pp}$.

Bendat, J. S. and A. G. Piersol, 1971: Random Data: Analysis and Measurement Procedures. Wiley-Interscience, New York, $407 \mathrm{pp}$.

Conne11, J. R., 1980: The Spectrum of Wind Speed Fluctuations Seen by a Rotating Blade of a Wind Energy Conversion System. Pacific Northwest Laboratory, Richland, Washington.

Etkin, B., 1961: Theory of the Flight of Airplanes in Isotropic TurbulenceReview and Extension. AGARD Report 372.

Lin, Y. K., 1967: Probabilistic Theory of Structural Dynamics. McGrawHi 11 Book Company, 366 pp.

Lumley, J. L., and H. A. Panofsky, 1964: The Structure of Atmospheric Turbulence. Wiley-Interscience, New York, $155 \mathrm{pp}$. 
APPENDIX A

APPROACH FOR DIRECT CALCULATION OF TWOTIME, TWO-POINT TURBULENCE STATISTICS 


\section{APPROACH FOR DIRECT CALCULATION OF TWO-}

IIME, TWO-POINT TURBULENCE STATISTICS

Consider a mean flow parallel to the $x_{1}$-axis with superimposed isotropic turbulence. We assume Taylor hypothes is is valid. Consider the two WECS blade elements \#1 and \#2 at distances $r_{1}$ and $r_{2}$ from the WECS hub and both rotating at angular rate $\omega_{0}$ as depicted in Figure 3 . We seek the covariance of the $i^{\text {th }}$ component of turbulence velocity at blade element $\# 1$ at time $t$ and the $j^{\text {th }}$ component of turbulence velocity at blade element $\# 2$ at time $t+\tau$. Now it can be shown that the Eulerian two-point covariance of $v_{j}\left(x_{1}+\xi_{1}\right.$, $\left.x_{2}+\xi_{2}, x_{3}+\xi_{3}, t+\tau\right)$ and $v_{j}^{\prime}\left(x_{1}, x_{2}, x_{3}, t\right)$ for homogeneous, isotropic turbulence is given by

$$
\begin{gathered}
R_{v_{i} v_{j}} \cdot\left(\xi_{1}-u_{0} \tau, \xi_{1}, \xi_{2}\right)=\sigma^{2} \quad\left\{[f(\xi)-g(\xi)\} \frac{\left(\xi_{j}-\delta_{1 j} u_{0} \tau\right)\left(\xi_{j}-\delta_{1 j} u_{0} \tau\right)}{\xi^{2}}+\right. \\
\left.g(\xi) \delta_{i j}\right\}
\end{gathered}
$$

where

$$
\xi=\left[\left(\xi_{1}-u_{0} \tau\right)^{2}+\xi_{2}^{2}+\xi_{3}^{2}\right]^{\frac{3}{2}}
$$

and $f(\xi)$ and $g(\xi)$ is the longitudinal and lateral correlation function. For the points \#1 and \#2 on the WECS

$$
\begin{gathered}
\xi_{1}=0 \\
\xi_{2}=r_{2} \cos \left(\omega_{0} t+\omega_{0} \tau+\alpha_{2}\right)-r_{1} \cos \left(\omega_{0} t+\alpha_{1}\right) \\
\xi_{3}=r_{2} \sin \left(\omega_{0} t+\omega_{0} \tau+\alpha_{2}\right)-r_{1} \sin \left(\omega_{0} t+\alpha_{1}\right)
\end{gathered}
$$

and

$$
\xi=\left\{u_{0}^{2} \tau^{2}+r_{1}^{2}+r_{2}^{2}-2 r_{1} r_{2} \cos \left(2 \omega_{0} t+\omega_{0} \tau+\alpha_{1}+\alpha_{2}\right)\right\}^{\frac{3}{2}} .
$$


Substitution of $(A-3)$ through $(A-6)$ into $(A-1)$ yields

$$
\begin{gathered}
R_{v_{i} v_{j}}\left(\tau ; t, r_{1}, r_{2}, \alpha_{1}, \alpha_{2}\right)=F_{i j}\left(\tau ; t, r_{1}, r_{2}, \alpha_{1}, \alpha_{2}\right)= \\
\left.R_{v_{i}^{\prime} v_{j}^{\prime}\left(-u_{0} \tau, \xi_{2}\right.}\left(\tau ; t, r_{1}, r_{2}, \alpha_{1}, \alpha_{2}\right), \xi_{3}\left(\tau ; t, r_{1}, r_{2}, \alpha_{1}, \alpha_{2}\right)\right)
\end{gathered}
$$

where $\xi_{2}$ and $\xi_{3}$ are functions of the indicated variables. The right side of Equation $(A-7)$ is the Eulerian two-point, two-time velocity component covariance function evaluated at points \#1 and \#2 on the WECS blade elements which corresponds to the two-point, two-time velocity component covariance function following the WECS blade elements. Specification of the longitudinal and lateral correlation function completely specifies $R_{v_{i} v_{j}}\left(\tau ; t, r_{1}, r_{2}, \alpha_{1}, \alpha_{2}\right)$.

The generalized power spectral density function corresponding to the two-point, two-time covariance function following the blade elements can be calculated with

$$
\Psi_{v_{i} v_{j}}\left(\omega_{1}, \omega_{2}\right)=\frac{1}{(2 \pi)^{2}} \int_{-\infty}^{\infty} F_{i j}(\tau ; t) e^{i\left(\omega_{1}-\omega_{2}\right) t} e^{-i \omega_{2} \tau} d t d \tau
$$

The corresponding frequency-time spectral density function is given by

$$
\Phi v_{i} v_{j}(\omega, t)=\int_{-\infty}^{\infty} \Psi_{i} v_{j}\left(\omega_{1}, \omega\right) e^{-i\left(\omega_{1}-\omega\right) t} d \omega_{1}
$$

It is understood that $F_{i j}, \Psi_{v_{j} v_{j}}$ and $\Phi_{v_{i} v_{j}}$ depend on $r_{1}, r_{2}, \alpha_{1}$ and $\alpha_{2}$.

Selection of reasonable functional forms of $f(\xi)$ and $g(\xi)$ for atmospheric turbulence (e.g., Dryden or von Karman models) do not lead to closed form integrals for Equations $(A-8)$ and $(A-9)$, thus, requiring numerical integration of these Fourier integrals. If we require generalized power spectral density and frequency-time spectral density functions for a relatively large number of points (e.g., $r_{1}, r_{2}, \alpha_{1}, \alpha_{2}$ ) then the engineering 
implementation of Equations $(A-8)$ and $(A-9)$ could be rather complicated. The virtue of using the Taylor series of the wind field about the hub and truncating third-and higher-order terms as discussed in the main body of this report is that one obtains closed forms for these functions in terms of $r_{1}, r_{2}, \alpha_{1}$ and $\alpha_{2}$. However, it should be noted that Equation (A-7) for the covariance function is simpler to implement in comparison to Equations (15) through (17) because numerical integration is required to obtain the back-transforms of the covariance on the right sides of Equations (16) and (17). 



\section{APPENDIX B \\ FORMULATION OF THEORY FOR NONHOMOGENEOUS TURBULENT FIELD}


TURBULENT FIELD

In this appendix we develop a procedure for calculating the velocity component covariance function following the WECS blade elements for a horizontally and statistically stationary boundary layer turbulent flow. The formulation in the section entitled COVARIANCES for the turbulence covariance following the WECS blade elements is general and thus can be applied to a vertically nonhomogeneous boundary layer. We seek the covariances on the rightsides of Equations (16) and (17). The formulation herein follows along the lines in the section entitled HOMOGENEOUS AND ISOTROPIC TURBULENCE. The two-fold wave number-frequency decomposition of $v_{i}^{\prime}\left(x_{1}, x_{2}, x_{3}, t\right)$ is given by

$$
v_{i}^{\prime}\left(x_{1}, x_{2}, x_{3}, t\right)=\int_{-\infty}^{\infty} e^{i\left(\kappa_{1} k_{1}+\omega t\right)} d z_{j}\left(\kappa_{1}, \kappa_{2}, x_{3}, \omega\right)
$$

where the repeated subscripts denote Einstein summation from 1 to 2 . We define

$$
\frac{\partial^{\nu+\lambda} v_{i}^{\prime}}{\partial x_{k}^{\nu} \partial x_{m}^{\lambda}}=\int_{-\infty}^{\infty} e^{i\left(x_{1} \kappa_{1}+\omega t\right)} D_{k}^{\nu} D_{m}^{\lambda} d z_{i}\left(\kappa_{1}, \kappa_{2}, x_{3}, \omega\right)
$$

(3)

where

$$
\begin{aligned}
& D_{2}=i k_{2} \\
& D_{3}=\frac{\partial}{\partial x_{3}} .
\end{aligned}
$$

Let

$$
\xi_{i k m \nu \lambda}(t)=\left(\frac{\partial^{\nu+\lambda} v_{j}^{\prime}}{\partial x_{k}^{\nu} \partial x_{m}^{\lambda}}\right)_{0}
$$


where subscript zero denotes evaluation at the WECS hub, so that

$$
\xi_{i k m u \lambda}(t)=\int_{-\infty}^{\infty} e^{i \omega t}\left[D_{k}^{v} D_{m}^{\lambda} d z_{i}\left(\kappa_{1}, k_{2}, x_{3}, \omega\right)\right]_{0}
$$

where Einstein summation convention does not apply to Greek subscripts. We construct the following covariance function

$$
\begin{gathered}
R_{\xi_{i k m \nu \lambda} \xi_{j s n \beta \gamma}}(\tau)=\int_{-\infty}^{\infty} e^{i \omega \tau} D_{k}^{\nu} D_{m}^{\lambda}\left(D_{s}^{-\beta}\right)^{*}\left(D_{n}^{-\gamma}\right)^{*} . \\
{\left[\left\langle d z_{i}\left(k_{1}, k_{2}, x_{3}, \omega\right) d z_{j}^{*}\left(k_{1}^{-}, k_{2}^{-}, x_{3}^{\prime}, \omega^{-}\right)\right\rangle\right]_{0,0} e^{i\left(\omega-\omega^{\prime}\right) t}}
\end{gathered}
$$

where

$$
\begin{aligned}
& D_{2}^{\prime}=i_{k_{2}^{\prime}}^{\prime} \\
& D_{3}=\frac{\partial}{\partial x_{3}^{\prime}}
\end{aligned}
$$

and double subscript denotes evaluation at the WECS hub with respect to $x_{3}$ and $x_{3}$. Invoking the conditions of statistical horizontal homogeneity and stationary yields

$$
\begin{gathered}
R_{\xi_{i k m \nu \lambda} \xi_{j s n \beta} \gamma}(\tau)=\int_{-\infty}^{\infty} e^{i \omega \tau}\left[D_{k}^{\nu} D_{m}^{\lambda}\left(D^{-\beta}\right)^{*}\left(D^{-\gamma}\right)^{*} .\right. \\
\left.\phi_{i j}\left(\kappa_{1}, \kappa_{2}, \omega ; x_{3}, x_{3}^{\prime}\right)\right]_{0,0} d \kappa_{1} d k_{2} d \omega
\end{gathered}
$$

where $\phi_{i j}\left(\kappa_{1}, k_{2}, w ; x_{3}, x_{3}\right)$ is the two-point, two-fold wave number-frequency spectral density of the functions $v_{i}^{\prime}\left(x_{1}, x_{2}, x_{3}, t\right)$ and $v_{j}^{\prime}\left(x_{1}, x_{2}, x_{3}^{\prime}, t\right)$. 
Application of Taylor's hypothes is permits us to write

$$
\phi_{i j}\left(\kappa_{1}, \kappa_{2}, x_{3}, \omega\right)=\phi_{i j}\left(\kappa_{1}, \kappa_{2}, x_{3}\right) \delta\left(\kappa_{1} u_{0}-\omega\right)
$$

so that Equation (B-10) can be written as

$$
R_{\xi_{j k m \nu \lambda} \xi_{j s n \beta \nu}}(\tau)=\int_{-\infty}^{\infty} e^{i \omega \tau} \xi_{j k m \nu \lambda} \xi_{j s n \beta \gamma}(\omega) d \omega
$$

where

$$
\begin{gathered}
{ }_{\xi_{j k m \nu \lambda} \xi_{j s n \beta \gamma}}(\omega)=\frac{1}{u_{0}} \int_{-\infty}^{\infty}\left[D_{k}^{v} D_{m}^{\lambda}\left(D_{s}^{\beta}\right)^{*}\left(D_{n}^{\gamma}\right)^{*} .\right. \\
\left.\phi_{i j}\left(\frac{\omega}{u_{0}}, \kappa_{2} ; x_{3}, x_{3}\right)\right]_{0,0} d \kappa_{2} .
\end{gathered}
$$

A11 of the necessary covariance functions on the right-sides of Equations (16) and (17) can be derived from Equations (B-12) and (B-13) upon specification of $\phi_{i j}\left(\frac{\omega}{u_{0}}, k_{2} ; x_{3}, x_{3}\right)$. The necessary $\psi$-functions in terms of $\phi_{i j}\left(\frac{\omega}{u_{0}}, k_{2} ; x_{3}, x_{3}\right)$ are given by

$$
\begin{aligned}
& \Psi_{w_{j} w_{j}}(\omega)=\frac{1}{u_{0}} \int_{-\infty}^{\infty} \phi_{i j}\left(\frac{\omega}{u_{0}}, k_{2}, 0,0\right) d k_{2} \\
& { }^{w} w_{i} p_{j}(\omega)=-\psi_{p_{j}} w_{j}(\omega)=-\frac{i}{u_{0}} \int_{-\infty}^{\infty} \kappa_{2} \phi i j\left(\frac{\omega}{u_{0}}, k_{2}, 0,0\right) d \kappa_{2} \\
& { }^{\Psi} w_{i} q_{j}(\omega)=\frac{1}{u_{0}}\left[\frac{\partial}{\partial x_{3}^{\prime}} \int_{-\infty}^{\infty} \phi_{i j}\left(\frac{\omega}{u_{0}}, k_{2} ; 0, x_{3}^{\prime}\right) d k_{2}\right]_{0} \\
& \psi_{i j} w_{j}(\omega)=\frac{1}{u_{0}}\left[\frac{\partial}{\partial x_{3}} \int_{-\infty}^{\infty} \phi_{i j}\left(\frac{\omega}{u_{0}}, k_{2} ; x_{3}, 0\right) d k_{2}\right]_{0}
\end{aligned}
$$




$$
\begin{aligned}
& \Psi_{w_{i} P_{j}}(\omega)=\Psi_{P_{i} w_{j}}(\omega)=-\frac{1}{u_{0}} \int_{-\infty}^{\infty} \kappa_{2}^{2} \phi_{i j}\left(\frac{\omega}{u_{0}}, k_{2}, 0,0\right) d_{k_{2}} \\
& \Psi_{w_{i} Q_{j}}(\omega)=\frac{1}{u_{0}}\left[\frac{\partial^{2}}{\partial x_{3}^{\prime 2}} \int_{-\infty}^{\infty} \phi_{i j}\left(\frac{\omega}{u_{0}}, k_{2} ; x_{3}, x_{3}\right) d k_{2}\right]_{0,0} \\
& \Psi_{Q_{i} w_{j}}(\omega)=\frac{1}{u_{0}}\left[\frac{\partial^{2}}{\partial x_{3}^{2}} \int_{-\infty}^{\infty} \phi_{i j}\left(\frac{\omega}{u_{0}}, \kappa_{2} ; x_{3}, x_{3}\right) d k_{2}\right]_{0,0} \\
& \Psi_{p_{i} p_{j}}(\omega)=-\Psi_{w_{i} P_{j}}(\omega) \\
& \psi_{q_{i} q_{j}}(\omega)=\frac{1}{u_{0}}\left[\frac{\partial^{2}}{\partial x_{3} \partial x_{3}^{\prime}} \int_{-\infty}^{\infty} \Phi_{i j}\left(\frac{\omega}{u_{0}}, k_{2} ; x_{3}, x_{3}^{\prime}\right) d k_{2}\right]_{0,0} \\
& \Psi_{w_{i} M_{j}}(\omega)=-\frac{i}{u_{0}}\left[\frac{\partial}{\partial x_{3}^{\prime}} \int_{-\infty}^{\infty} \kappa_{2} \phi_{i j}\left(\frac{\omega}{u_{0}}, \kappa_{2} ; 0, x_{3}\right) d_{\kappa_{2}}\right]_{0} \\
& \Psi_{M_{i} w_{j}}(\omega)=-\frac{i}{u_{0}}\left[\frac{\partial}{\partial x_{3}} \int_{-\infty}^{\infty} k_{2} \phi_{i j}\left(\frac{\omega}{u_{0}}, k_{2}, x_{3}, 0\right) d_{k_{2}}\right]_{0}
\end{aligned}
$$




\section{APPENDIX C}

SPECTRAL CONTRIBUTION RESULTING FROM MEAN FLOW SHEAR 
The presence of a mean flow wind shear contributes to the turbulence spectrum for $i=j=1$. In the most strict sense the theory presented herein does not permit this contribution because the theory requires the mean flow wind profile to be a constant. However, we shall allow the mean flow to vary with height above natural grade to calculate the contribution of mean flow wind shear to the $i=j=1$ spectral density function. We expand the mean wind profile about the hub, so that

$$
\bar{u}\left(x_{3}\right)=u_{0}+\left(\frac{\partial \bar{u}}{\partial x_{3}}\right)_{0} x_{3}
$$

where we have truncated second- and higher-order terms. The fluctuations in the mean flow relative to the constant wind profile $u_{0}$ as seen by a WECS blade element are thus given by

$$
\bar{u}^{\prime}\left(x_{3}\right)=\left(\frac{\partial \bar{u}}{\partial x_{3}}\right)_{0} r \sin \left(\omega_{0} t+\alpha\right) .
$$

Evaluation of Equation $(C-2)$ at time $t+\tau$ at blade element located at $\left(r_{1}, \alpha_{1}\right)$ and at time $t$ at blade element located at $\left(r_{2}, \alpha_{2}\right)$, multiplying the resulting relationships, and ensemble averaging yields

$$
\left\langle\bar{u}^{\prime}\left(t, \tau ; r_{1}, \alpha_{1}\right) \bar{u}^{\prime}\left(t, 0 ; r_{2}, \alpha_{2}\right)\right\rangle=R_{\bar{u}}\left(t, \tau ; r_{1}, r_{2}, \alpha_{1}, \alpha_{2}\right)
$$

where

$$
\begin{gathered}
R_{\bar{u}}\left(t, \tau ; r_{1}, r_{2}, \alpha_{1}, \alpha_{2}\right)=\left(\frac{\partial \bar{u}}{\partial x_{3}}\right)_{0}^{2} \frac{r_{1} r_{2}}{2}\left[\cos \left(\omega_{0} \tau+\alpha_{2}-\alpha_{1}\right)\right. \\
\left.-\cos \left(\omega_{0}(2 t+\tau)+\alpha_{1}+\alpha_{2}\right)\right] .
\end{gathered}
$$

The first term on the right in the brackets is the stationary contribution to 
the covariance and the second term is the nonstationary contribution. For the logarithmic profile

$$
\left(\frac{\partial \bar{u}}{\partial z}\right)_{0}=\frac{u_{\star_{0}}}{k z_{h}}
$$

where

$$
\begin{aligned}
& u_{\star 0}=\text { surface friction velocity } \\
& k=\text { von Karman's constant }
\end{aligned}
$$

(Lumley and Panofsky, 1964), so that the coefficient of the trigonometric terms in Equation $(\mathrm{C}-4)$ given by

$$
\left(\frac{\partial \bar{u}}{\partial x_{3}}\right)_{0}^{2} \frac{r_{1} r_{2}}{2}=\frac{u_{\star 0}^{2}}{k^{2} z_{h}^{2}} \frac{r_{1} r_{2}}{2} .
$$

The double frequency generalized power spectral density function associated with Equation $(C-4)$ is given by

$$
\begin{gathered}
\Psi_{\bar{u}}\left(\omega_{1}, \omega_{2} ; r_{1}, r_{2}, \alpha_{1}, \alpha_{2}\right)=\left(\frac{\partial \bar{u}^{2}}{\partial x_{3}}\right)_{0}^{2} \frac{r_{1} r_{2}}{2}\left\{\left[e^{i\left(\alpha_{2}-\alpha_{1}\right)} \delta\left(\omega_{1}+\omega_{0}\right)\right.\right. \\
+e^{\left.-i\left(\alpha_{2}-\alpha_{1}\right) \delta\left(\omega_{1}-\omega_{0}\right)\right] \delta\left(\omega_{1}-\omega_{2}\right)-\left[e^{i\left(\alpha_{1}+\alpha_{2}\right)} \delta\left(\omega_{1}-\omega_{2}+2 \omega_{0}\right) \delta\left(\omega_{1}+\omega_{0}\right)\right.} \\
+e^{\left.\left.-i\left(\alpha_{1}+\alpha_{2}\right) \delta\left(\omega_{1}-\omega_{2}-2 \omega_{0}\right) \delta\left(\omega_{1}-\omega_{0}\right)\right]\right\}}
\end{gathered}
$$

The frequency-time spectral density function is given by

$$
\begin{aligned}
& \Phi_{\bar{u}}\left(\omega, t ; r_{1}, r_{2}, \alpha_{1}, \alpha_{2}\right)=\left(\frac{\partial \bar{u}}{\partial x_{3}}\right)_{0}^{2} \frac{r_{1} r_{2}}{4}\left\{e^{i\left(\alpha_{2}-\alpha_{1}\right)} \delta\left(\omega+\omega_{0}\right)+e^{-i\left(\alpha_{2}-\alpha_{1}\right)} \delta\left(\omega-\omega_{0}\right)\right. \\
& \left.-\left[e^{i\left(\alpha_{1}+\alpha_{2}\right)} e^{i\left(\omega+\omega_{0}\right) t} \delta\left(\omega-\omega_{0}\right)+e^{-i\left(\alpha_{1}+\alpha_{2}\right)} e^{i\left(\omega-\omega_{0}\right) t_{\delta}\left(\omega+\omega_{0}\right)}\right]\right\} \cdot(c-8)
\end{aligned}
$$

The first two terms in the curly brackets on the right side of Equation (C-8) corresponds to the stationary contribution to the spectrum while the remaining terms correspond to nonstationary contribution. Integration of Equation ( $C-8)$ over the domain $-\infty<\omega<\infty$ yields the variance of $\vec{u}$, Equation $(C-4)$ evaluated at $\tau=0$. 


\section{DISTRIBUTION}

No. of

Copies

OFFSITE

J. Cadogan

Department of Energy

Wind Energy Technology Division

1000 Independence Avenue

Forresta 1 Building, Room $5 \mathrm{HO} 48$

Washington, DC 20585

L. V. Divone

Department of Energy

Wind Energy Technology Division

1000 Independence Avenue

Forrestal Building, Room $5 \mathrm{HO} 48$

Washington, DC 20585

G. P. Tennyson

Department of Energy

Albuquerque Operations Office 4501 Indian School Road N.E.

Albuquerque, NM 87110

27 DOE Technical Information Center

Peter Lissaman

Aerovironment, Inc.

145 Vista Avenue

Pasadena, CA 91107

D. K. Ai

Alcoa Laboratories

Alcoa Technical Center

Alcoa Center, PA 15069

Tom Gray

American Wind Energy Association 2010 Massachusetts Avenue

Fourth Floor

Washington, DC 20036

W. A. Vachon

Arthur D. Little, Inc.

20 Acorn Park

Cambridge, MA 02140
No. of

Copies

Don McGrew

Boeing Engineering and Construction

P.0. Box 3707, Mail Stop 9A-67

Seattle, WA 98124

S. J. Hightower

Bureau of Reclamation

Denver Federal Center

Building 67, Code 254

Denver, C0 80225

Joe Hennessy

Wind Energy Program

California Energy Commission

1516 9th Street

Sacramento, CA 95814

Professor Arnold Court

Department of Geography

California State University

Northridge, CA 91130

R. N. Meroney

Department of Civil Engineering

Colorado State University

Fort Collins, CO 80523

James Telford

Desert Research Institute

Atmospheric Sciences Center

University of Nevada System

P.0. Box 60220

Reno, NV 89506

2 Dr. Edgar DeMeo/Frank Goodman, Jr. Electric Power Research Institute 3412 Hillview Avenue

Palo Alto, CA 94303

George Ficht

7703 Oakridge Drive

Huntsville, AL 35802 
No. of

Copies

2 Tom Hiester/J. J. Riley

Flow Wind Corporation 21414-68th Avenue South

Kent, WA 98031

Walter Frost

FWG Associates, Inc.

271A Lakewood Drive

Tullahoma, TN 37388

Robert Barton

Advanced Energy Power Division

General Electric Company

Mail Stop 7310

501 Al lendale Road, P.0. Box 527

King of Prussia, PA 19406

Art Jackson

Hamilton Standard Division United Technologies Corporation Windsor Lock, CT 06096

2 J. M. Kos/Eugene Di Valentin Hamilton Standard Bradley Field Road windsor Locks, CT 06096

M. K. Goldenblatt

JBF Scientific Corporation 2 Jewel Drive

Wilmington, MA 01887

M. A. Bowes

Kaman Aerospace Corporation

01d Windsor Road

Bloomfield, CT 06095

G. L. Park

Department of Electrical Engineering

Michigan State University

East Lansing, MI 48824

J. Konigsberg

Montana Energy Office

Capital Station

Helena, MT 59601
No. of

Copies

David Spera

NASA/Lewis Research Center

21000 Brookpark Road

Cleveland, $\mathrm{OH} 44135$

Wind Turbine Group

NASA/Lewis Research Center

21000 Brookpark Road

Cleveland, $\mathrm{OH} 44135$

3 Phillip French

NASA Scientific and Technical Information Facility

P.0. Box 8757

Baltimore/Washington International Airport

Baltimore, MD 21240

2 J. C. Kaimal/W. Hooke

National Oceanic and Atmospheric Administration/Environmenta 1 Research Laboratories/Wave Propagation Laboratory

3000 Marine Street

Boulder, CO 80302

Peter M. Moretti

Oklahoma State University

Mechanical and Aerospace Engineering

Engineering North 218

Stillwater, OK 74074

2 W. Holley/R. Thresher

Department of Mechanical Engr.

Oregon State University

Corvallis, OR 97331

Don Bain

Department of Energy

State of Oregon

Labor and Industries Building

Room 111

Salem, OR 98310

R. Lee Cresap

Pacific Gas \& Electric Company

Room 1385

77 Beale Street

San Francisco, CA 94106 
No. of

Copies

R. L. Simon

Pacific Gas and Electric

215 Market Street

San Francisco, CA 94106

2 J. Dutton/H. A. Panofsky

Department of Meteorology

Pennsylvania State University

University Park, PA 16902

3 Ernel L. Luther

Planning Research Corporation 1500 Planning Research Drive

McLean, VA 22102

J. P. Sullivan

331 Grissom Hall

Purdue University

West Lafayette, IN 47907

Terry J. Healy

Rockwell International

Rocky Flats Plant

P.0. Box 464

Golden, C0 80401

E. Kadlec

Sandia Laboratories

Division 5443, P. 0 . Box 5800

Albuquerque, NM 87115

Robert Noun

Solar Energy Research Institute

1617 Cole Boulevard

Golden, Co 80401

Bruce Bailey

Atmospheric Sciences Research Center

State University of New York at Albany

Albany, NY 12222

Sherman M. Chan

Systems Control, Inc.

1801 Page Mill Road

P.0. Box 10025

Palo Alto, CA 94303
No. of

Copies

W. Owens

Tennessee Valley Authority

320 Credit Union Building

715 Market Street

Chattanooga, TN 37401

R. H. Kirchhoff

Department of Mechanical Engr. University of Massachusetts

Amherst, MA 01003

Earl L. Davis

U.S. Windpower, Inc.

6421 B. South Front Road

Livermore, CA 94550

2 R. E. Akins/H. Tieleman

Department of Engineering Science \& Mechanics

Virginia Polytechnic Institute and State University

Blacksburg, VA 24061

Susan Hosch

Washington State Energy Office

$400 \mathrm{E}$. Union Avenue, 1st Floor

Olympia, WA 98504

T. Anderson

Westinghouse Electric Co.

P.0. Box 10824

Pittsburgh, PA 15236

G. D. Thomann

Wichita State University

P. 0. Box 44

Wichita, KS 67208

Farrell Smith Seiler

Wind Energy Report

Box 14 - 104 S. Village Avenue

Rockville Centre, NY 11571

Dr. V. Barros

28 De Julio 28

9120 Puerto Madryn

Chulret R. ARGENTINA 
No. of

Copies

Leif Kristensen

Department of Physics

Ris $\emptyset$ National Laboratory

4000 Roskilde

DENMARK

Per Lundsager

Ris $\emptyset$ National Laboratory

P. 0. Box 49

DK-4000 Roskilde

DENMARK

Professor Sir Martin Ryle

Cavendish Laboratory

Madingley Road

Cambridge CB3 OHE

ENGLAND

H. H. Rosenbrock

The University of Manchester

Institute of Science and Technology

P.0. Box No. 88

Manchester M60 1QD

ENGLAND

D. Lindley

Taylor Woodrow Construction, Ltd.

Taywood House

345 Ruislip Road

Southal 1

Middlesex UBI 2QX

ENGLAND

Dr. Werner Dub

Forschungsprojekt Windenergie

Universitätsstrasse 31

D-8400 Regensburg

GERMANY
No. of

Copies

Dr. Neil Cherry

Lincoln College

Canterbury

NEW ZEALAND

Dr. 011e Ljungstrom

FFA, The Aeronautical Research Institute

Forskningsstationen i Stockholm

Drottning Kristinas Vag 47

S-114 29 Stockholm

SWEDEN

ONSITE

DOE Richland Operations Office

H. E. Ransom

16 Pacific Northwest Laboratory

J. R. Connell

C. E. Elderkin

A. H. Miller

D. C. Powel1

Technical Information Files (5)

Publishing Coordination (2)

WCPE Program Office (5) 


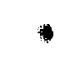

.

*

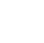

